\title{
The Canadian
}

Journal of Statistics

La revue canadienne

de statistique

Time Series, Point Processes, and Hybrids

Author(s): David R. Brillinger

Reviewed work(s):

Source: The Canadian Journal of Statistics / La Revue Canadienne de Statistique, Vol. 22, No. 2 (Jun., 1994), pp. 177-206

Published by: Statistical Society of Canada

Stable URL: http://www.jstor.org/stable/3315583

Accessed: 11/03/2013 19:48

Your use of the JSTOR archive indicates your acceptance of the Terms \& Conditions of Use, available at

http://www.jstor.org/page/info/about/policies/terms.jsp

JSTOR is a not-for-profit service that helps scholars, researchers, and students discover, use, and build upon a wide range of content in a trusted digital archive. We use information technology and tools to increase productivity and facilitate new forms of scholarship. For more information about JSTOR, please contact support@jstor.org. 


\title{
Time series, point processes, and hybrids*
}

\author{
David R. BRILLINGER
}

The University of California, Berkeley

Key words and phrases: Bispectrum, earthquake, marked point process, microtubule, motility, point process, seal, stacking, stationary increments, tide, time series. AMS 1985 subject classifications: Primary 60G12, 60G55, 60G57, 62M10, 62M15

\section{Dedicated to Dan DeLury, applied statistician extraordinaire.}

\begin{abstract}
Techniques developed for the study of time series, point processes, and marked point processes can suggest corresponding techniques for each other, and common techniques can be recognized. In this paper connections are drawn based on conceptual foundations, basic parameters, analyses, displays, algorithms, problems, models. The definitions and techniques are brought out by specific scientific problems. The emphasis is on the single-realization stationary case and on the use of second- and third-order moments to help understand the realization. The tool of stacking, at a particular period, is employed in several of the examples.
\end{abstract}

\section{RÉSUMÉ}

Les techniques développées pour l'étude des séries chronologiques, des processus ponctuels et des processus ponctuels marqués peuvent suggérer des techniques correspondantes pour chacun des autres problèmes, et des techniques communes peuvent être discernées. Dans cet article, nous établissons des correspondances fondées sur des bases conceptuelles, des paramètres fondamentaux, des analyses, des visualisations, des algorithmes, des problèmes et des modèles. Les définitions et techniques proviennent de problèmes scientifiques spécifiques. Nous insistons plus particulièrement sur le cas stationnaire de réalisation simple et l'utilisation des moments de second et de troisième ordre, afin de comprendre cette réalisation. Nous employons une technique d'empilage, à une période particulière, pour plusieurs exemples.

\section{INTRODUCTION}

Parallel analysis procedures are often available for time series, point processes, and marked point processes. An intention of this paper is to bring some of these parallels out. Each domain can learn from the others.

A time series $Y$ is a wiggly line $Y(t),-\infty<t<\infty$. A point process $N$ is a collection of times $\left\{\tau_{j}, j=0, \pm 1, \pm 2, \ldots\right\}$. (It will be assumed that the $\tau_{j}$ are distinct.) A marked point process $J$ is a collection of times and associated quantities (marks) $\left\{\left(\tau_{j}, M_{j}\right), j=0, \pm 1, \pm 2, \ldots\right\}$. There are also hybrids such as sampled time series $\left\{Y\left(\tau_{j}\right), j=0, \pm 1, \pm 2, \ldots\right\}$. Time-series techniques and time-series data are common. Point-process techniques are less common, as are their analyses. Studies of marked point

\footnotetext{
*This paper is based on the Gold Medal Lecture presented at the Annual Meeting of the Statistical Society of Canada held 6-9 June 1993 at Acadia University in Wolfville, Nova Scotia. This research was supported in part by NSF Grants DMS-9208683, DMS-9300002, and ONR Grant N00014-94-1-0042.
} 
processes are the rarest, but are under substantial current development, particularly for the spatial case.

This paper is partly expository, seeking to bring out connections amongst disparate processes, and partly a presentation of new techniques and analyses. It will be seen that the second- and third-order moments can be useful tools with which to address particular scientific problems of interest. Estimates of such moments and corresponding spectra are provided for some particular time-series, point-process, and marked-point-process data sets-specifically, ocean tides, nerve-cell firings, and earthquake occurrences. Section 4 lists some analytic methods useful for studying the processes. Section 5 describes two current investigations: analyzing the tracks of microtubules and the tracks of seals. The computational details are given in the Appendix.

\section{DISPLAYING PROCESS DATA}

\subsection{Time-Series Displays.}

The time-series plot is the most frequently used form of graphic design. (Tufte 1983)

With these words Tufte makes clear the dramatic importance of graphs of time series. He further presents a wide variety of specific examples. The book by Cleveland (1985) devotes a number of pages to the topic. Among the displays Cleveland mentions are the connected, symbol, connected symbol, and vertical-line displays.

The top display of Figure 1 provides a shaded area graph for the sea height at Saint John, New Brunswick. The heights are measured from a particular level called chart datum. Saint John is on the Bay of Fundy, whose tides are the largest in the world, reaching 17 meters in places. General discussions of the analysis of tides may be found in Morettin and de Mesquita (1978), Wood (1978), and Forrester (1983) for example.

Examination of Figure 1 suggests a phenomenon of frequency approximately 2 cycles per day. The technique of stacking is convenient for examining such a circumstance. One places successive segments of a series above each other. In the bottom display of Figure 1, 24-h segments have been stacked starting at times $0,24,48, \ldots \mathrm{h}$ after midnight 31 December 1990. The times of high tide are lagging slightly each day. The lag is caused by the fact that it takes the earth approximately $24 \mathrm{~h} 52 \mathrm{~min}$ to make one full rotation with respect to the moon.

Stacking is an important display procedure in seismology; see Waters (1978). It is particularly useful for series with strong periodicity. The original values are graphed; hence there are special opportunities for noticing unusual features. Stacking may be seen as a graphical form of the Buys-Ballot table (Buys-Ballot 1847). Suppose one has data $Y(t), t=0,1,2, \ldots$. In the Buys-Ballot table, to study the integer-valued period $P$, one creates a matrix with entry $Y((i-1) P+j)$ in row $i$, column $j$. Typically the columns means are computed and examined. A related table was used in Laplace (1825) to study the relationship of tides to equinoctial syzygies. The radar memory tube that was so important in World War II (see Watson-Watt 1946) can be thought of as a variant. Whittaker and Robinson (1944) and Brillinger (1974) suggest formal test procedures based on Buys-Ballot table values.

\subsection{Point Process Displays.}

There are several common methods for displaying point-process data. These include points on a line, step function, and lines on a line. 
Saint John Tides, January 1991

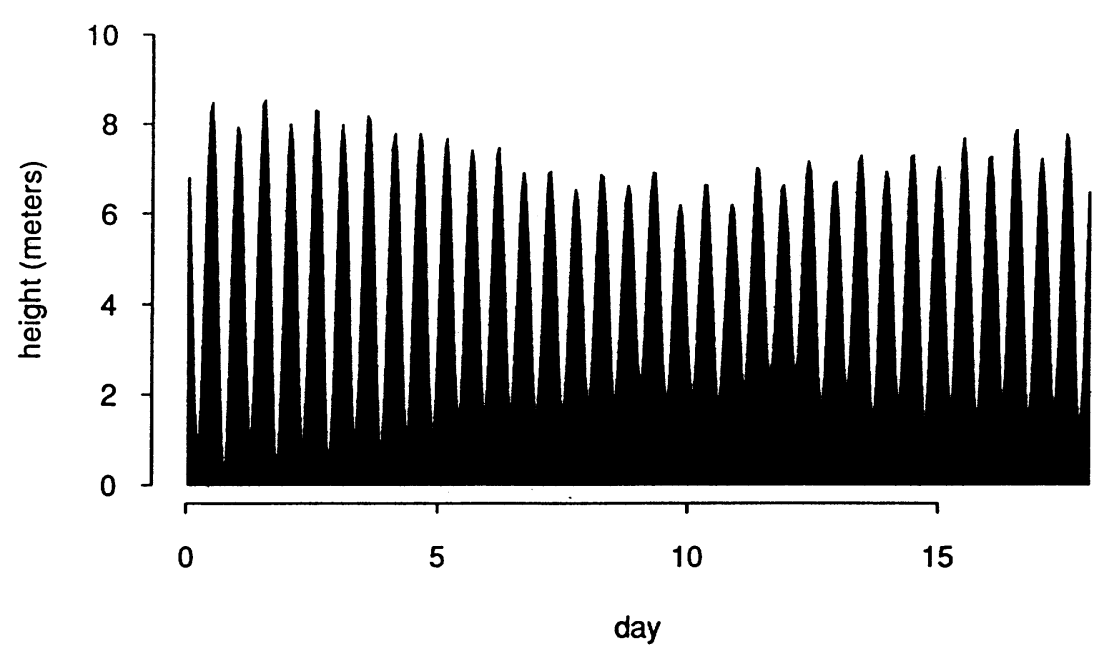

Stacked daily values

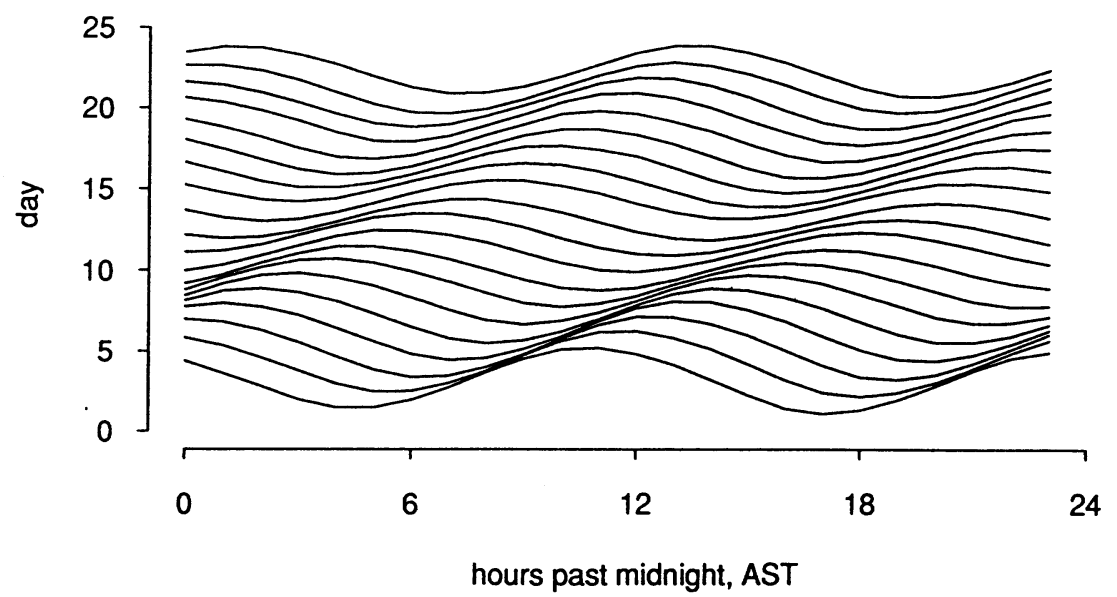

FIGURE 1: A graph of hourly tidal values at Saint John, New Brunswick for the period 1 to 18 January 1991. The lower graph stacks successive 24-hour segments above preceding ones. The $y$-axis labels the day of the segment.

Figure 2 presents point-process displays derived from some neurophysiological data. The top display is a recorded continuous time signal, the fluctuating voltage within a neuron. Spikes are seen to recur. These correspond to the times at which the neuron is firing and may be thought of as a realization of a point process. The middle display is a step function increasing by 1 at each firing time. The bottom display employs vertical lines to represent the firing times. The data are from the sea hare. The data collection is described in Bryant et al. (1973). 


\section{Transmembrane Potential, $\mathrm{Y}(\mathrm{t})$}

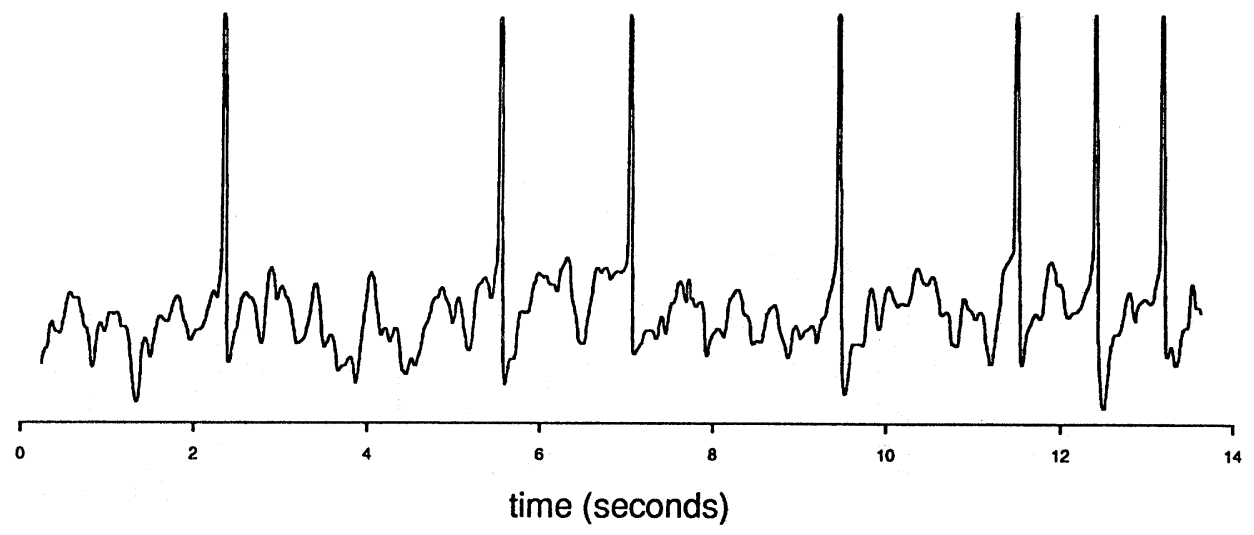

\section{$\mathrm{dN}(\mathrm{t}) / \mathrm{dt}$}
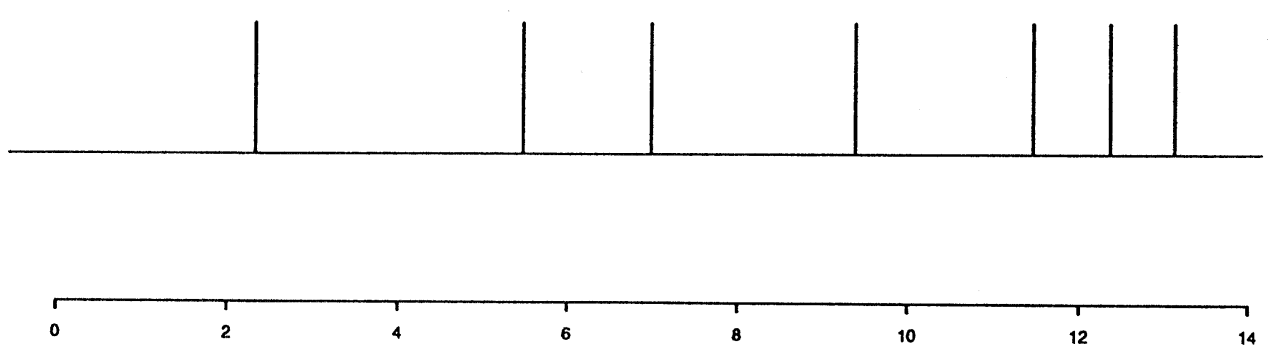

Figure 2: The top display is the fluctuating voltage potential within a neuron. The middle display is a step function increasing by 1 at the time of each spike in the top display. The bottom display has a vertical line at the location of each spike. The bottom two provide different methods of displaying point-process data. 


\section{Neuron L10 bursting with accelerando}
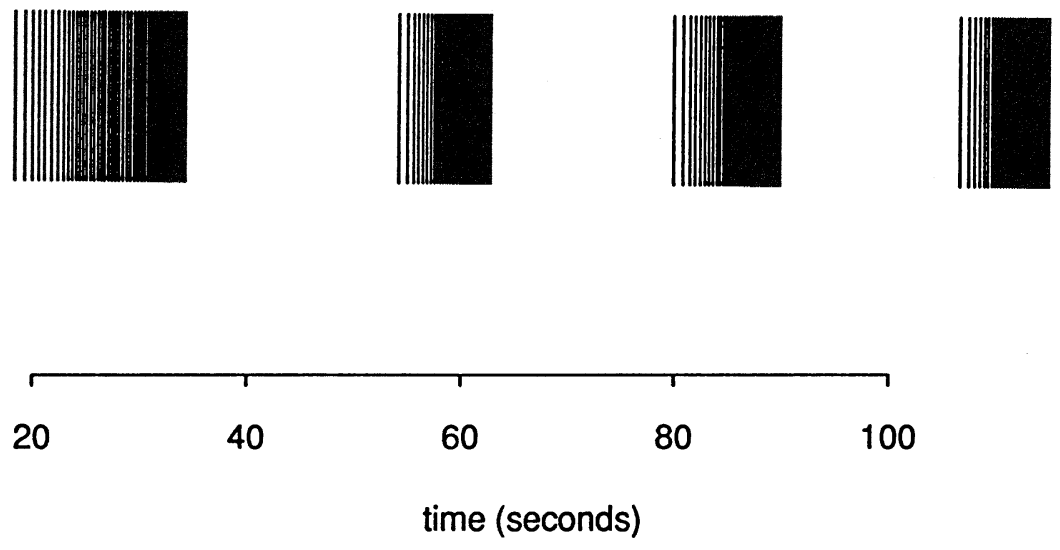

\section{Stacked bursts}

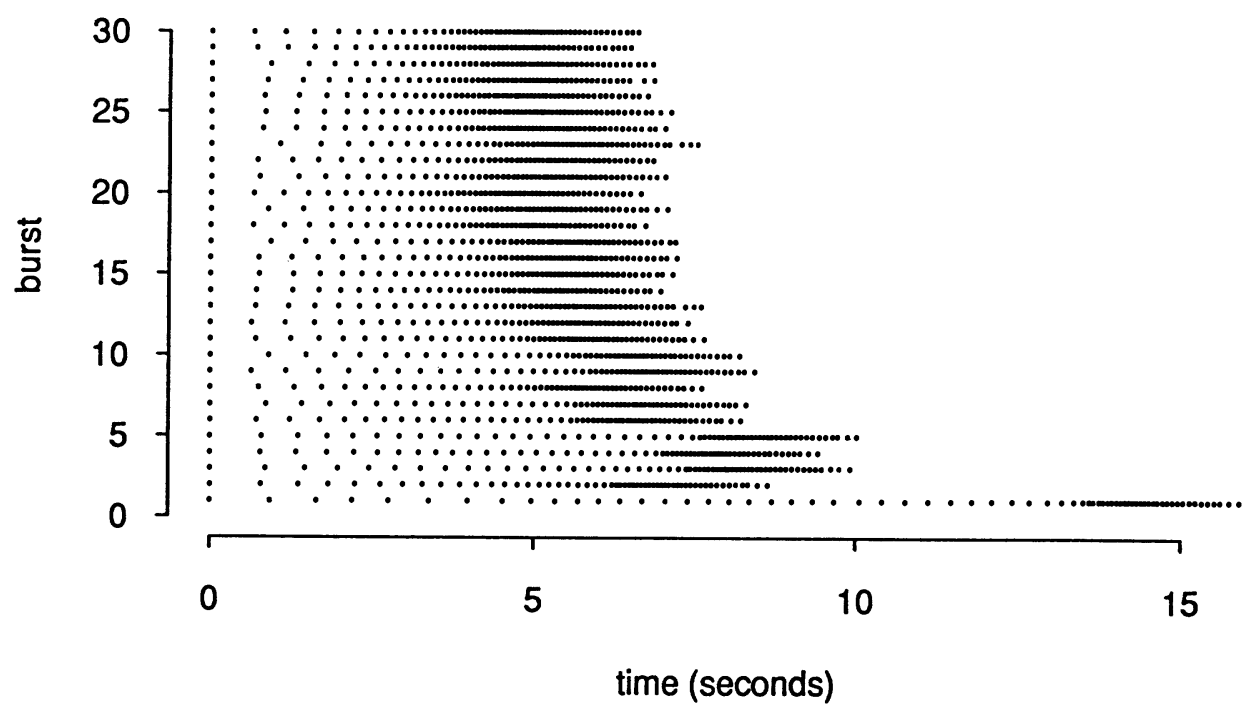

FIGURE 3: The firing of a neuron of the sea hare. The bottom display presents the times of the firings in each burst relative to the time of the first firing.

Figure 3 also presents spike-train data, this time for another neuron of the sea hare. The top display shows firing occurring in bursts, with vertical lines again corresponding to firing times. In order to better understand the structure of these data, a stacked plot was prepared. This is the bottom display of the figure. Each line starts with the time of the first spike of a burst. The other spikes of the burst then follow across the line. The display, in this case, brings out some nonstationarity in the data-the bursts are decreasing slowly in duration.

Cox and Lewis (1966, p. 14) plot the column means of a Buys-Ballot table for point-process data. A Fourier-transform-based test procedure for periodicities is given in Brillinger (1974). 


\subsection{Hybrid Process Displays.}

In the case that the marks of a marked point process are real-valued, the process is known as a jump process or cumulative process. The realizations may be represented by lines along a line. An example is the top display of Figure 4. This graphs the times and magnitudes of earthquakes of magnitude 5.0 or greater in California from 1932 to mid 1992.

A second method of display is via a cumulative function, summing the mark values up to time $t$. The bottom display presents such a plot for a quantity proportional to the amount of energy $e^{M}$ released in an earthquake, $M$ the magnitude. The dashed line is provided to allow some assessment of the stationarity of the process. A third plotting procedure, when the marks are velocities of vehicles passing a point, suggested by Bartlett (1967), is to plot fixed-length lines, starting at the time points, with slopes related to the mark values.

Figure 5 presents the earthquake data stacked by year. The times are the centers of the circles. The circles relate to the size of the event, with the scale indicated. Various researchers have conjectured that a yearly periodicity exists in the occurrence of earthquakes; see Davison (1928) and Katsumura (1985) for example. Figure 5 does not suggest the presence of such an effect in the present case.

\section{STATIONARY INCREMENT PROCESS}

In this section some specific processes are discussed. In the cases emphasized, each can be related to a process with stationary increments.

$X(\cdot)$ is called a process with stationary increments if the following holds: $X(t),-\infty<$ $t<\infty$, is such that the joint distribution of the increments $X\left(t+b_{1}\right)-X\left(t+a_{1}\right), \ldots, X(t+$ $\left.b_{k}\right)-X\left(t+a_{k}\right)$ does not depend on $t$ for any $a_{1}<b_{1}, \ldots, a_{k}<b_{k}$ and $k=1,2,3, \ldots$. The basic ideas are due to Kolmogorov and may be found on pp. 551-559 in Doob (1953). There exists a statistical calculus for such processes; see Brillinger (1972).

Next, it will be illustrated how the processes of Section 2 can be related to processes of stationary increments. A stationary time series $Y$ corresponds to a stationary increment process $X$ via

$$
X(t)=\int_{0}^{t} Y(u) d u
$$

A stationary point process $N$ corresponds to a stationary increment process $N(\cdot)$ in which all the increments $N(t+b)-N(t+a), a<b$, are nonnegative integers, specifically $N(t+b)-N(t+a)=\#\left\{\tau_{j} \mid t+a<\tau_{j} \leq t+b\right\}$. One can write

$$
X(t)=\int_{0}^{t} d N(u) .
$$

A marked point process $J$ with real-valued marks may be represented via $J(t)=$ $\sum_{0<\tau_{j} \leq t} M_{j}$, and there is the correspondence

$$
X(t)=\int_{0}^{t} d J(u) .
$$

Consider the case of a process $X$ with stationary increments, $\mathcal{E}\{d X(t)\}=c_{X} d t$ with $c_{X}$ the mean intensity. For simplicity suppose $c_{X}$ to be 0 . [If not, replace $X(t)$ by $X(t)-c_{X} t$.] One defines the autocovariance measure $C_{X X}$ via

$$
\mathcal{E}\{d X(t+u) d X(t)\}=d C_{X X}(u) d t,
$$




\section{California earthquakes}

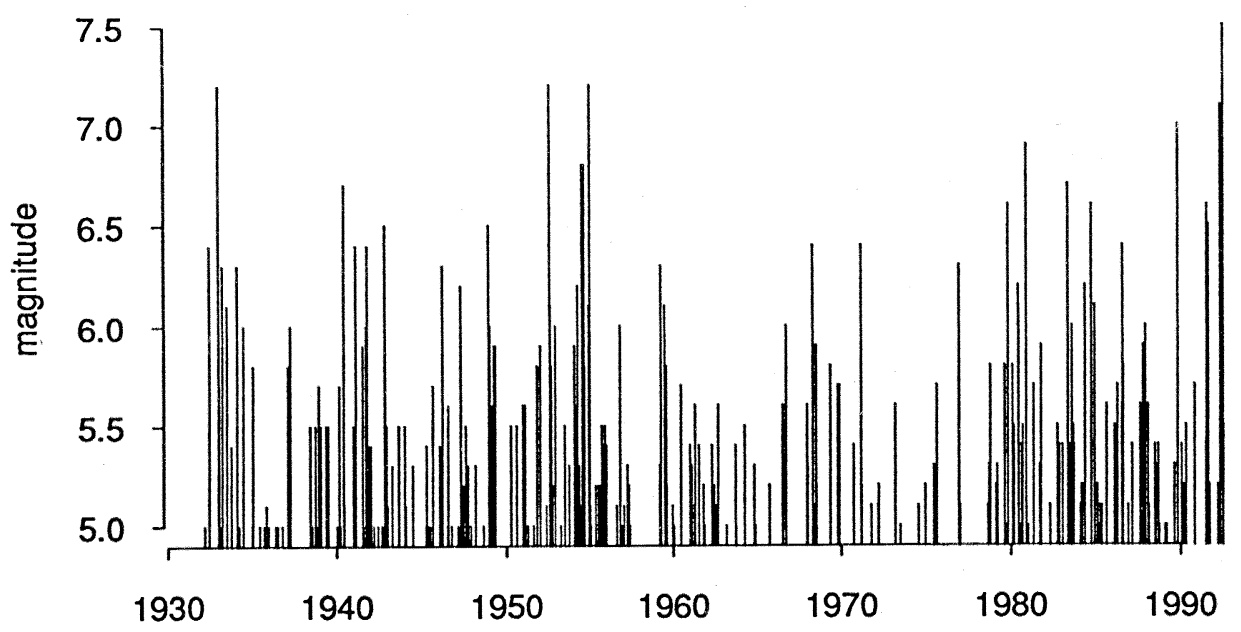

\section{Cumulative energy}

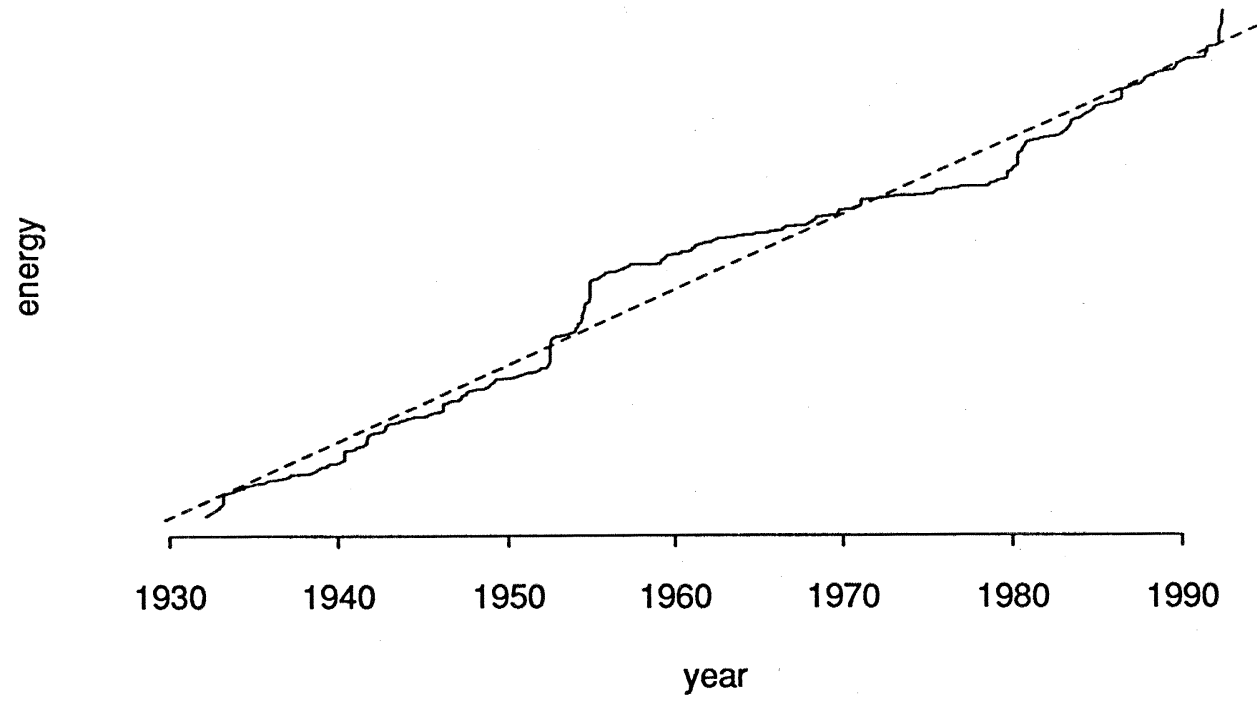

FIGURE 4: The top display indicates times of earthquakes and associated magnitudes. The events are for California, in the period 1932 to mid 1992 and for events of magnitude 5.0 or greater. The bottom display is proportional to the cumulative energy released by these events. 


\section{California events stacked by year}

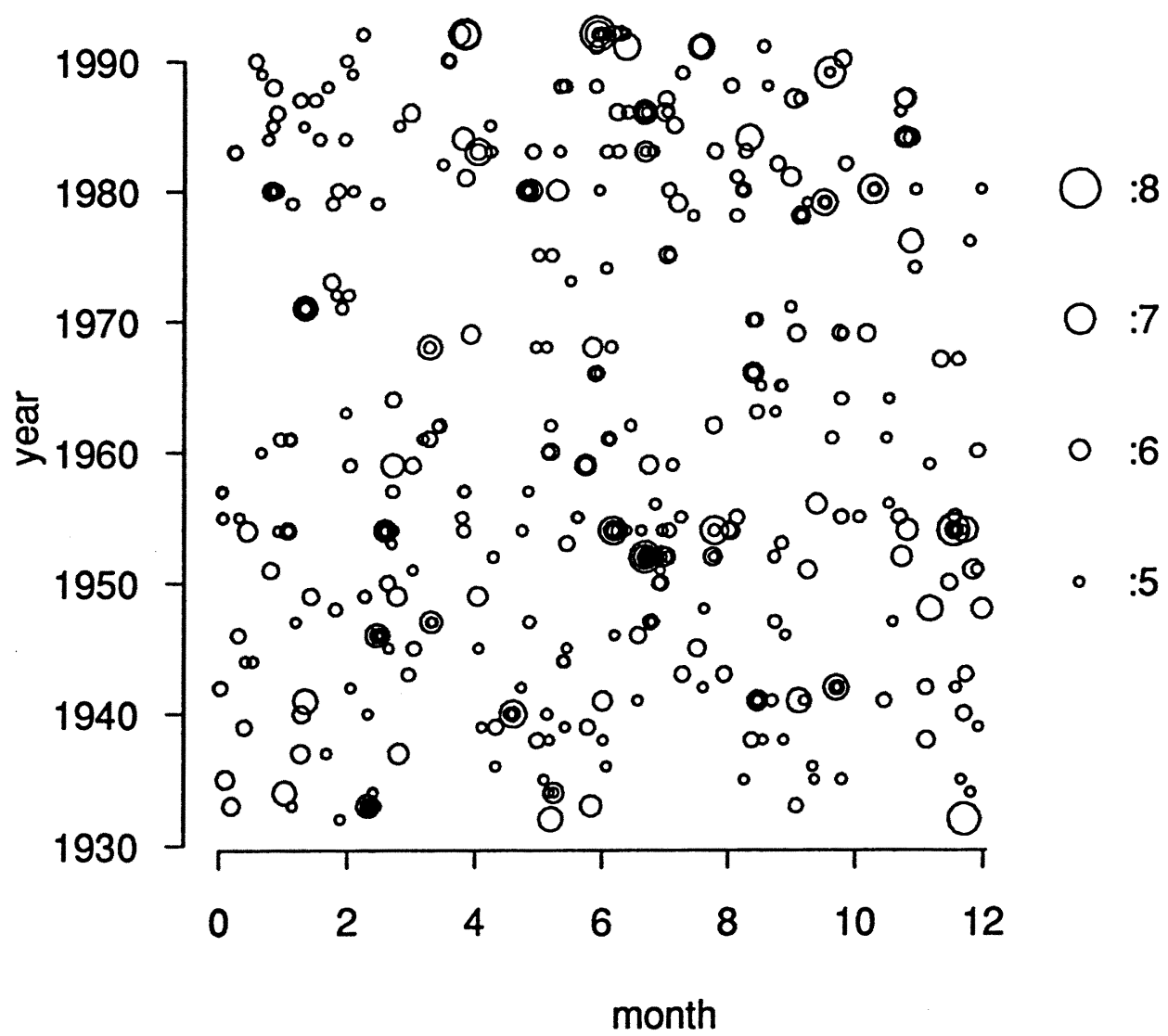

FIGURE 5: The events of Figure 4 stacked by year, with circle diameter indicating magnitude. The circles on the right indicate the correspondence between circle size and magnitude.

and the third cumulant measure $C_{X X X}$ via

$$
\mathcal{E}\{d X(t+u) d X(t+v) d X(t)\}=d C_{X X X}(u, v) d t .
$$

The process $X$ has a spectral representation

$$
X(t)=\int \frac{e^{i t \lambda}-1}{i \lambda} d Z(\lambda)
$$

with $Z$ a random function such that

$$
\mathcal{E}\{d Z(\lambda) d Z(\mu)\}=\delta(\lambda+\mu) f_{X X}(\lambda) d \lambda d \mu
$$

and

$$
\mathcal{E}\{d Z(\lambda) d Z(\mu) d Z(v)\}=\delta(\lambda+\mu+v) f_{X X X}(\lambda, \mu) d \lambda d \mu d v
$$


$\delta(\cdot)$ being the Dirac delta function and $f_{X X}, f_{X X X}$ the power spectrum and bispectrum respectively. These are Fourier-Stieltjes transforms of $C_{X X}, C_{X X X}$ respectively. The spectra themselves may be generalized functions containing Dirac deltas. [Details may be found in Brillinger (1972).]

\subsection{The Time-Series Case.}

Consider a zero-mean stationary time series $Y(t),-\infty<t<\infty$. Following (3.1)-(3.3), the autocovariance function is given by

$$
\mathcal{E}\{Y(t+u) Y(t)\}=\frac{d}{d u} C_{X X}(u)=c_{Y Y}(u),
$$

and the third-order cumulant function by

$$
\mathcal{E}\{Y(t+u) Y(t+v) Y(t)\}=\frac{\partial}{\partial u} \frac{\partial}{\partial v} C_{X X X}(u, v)=c_{Y Y Y}(u, v)
$$

The spectral representation is

$$
Y(t)=\int e^{i t \lambda} d Z(\lambda)
$$

with $Z$ satisfying (3.5) and (3.6).

The autocovariance function (3.7) provides a measure of the dependence of values of the series lag $u$ time units apart. An estimate is provided in Figure 6 for the Saint John tidal series. The data are for the time period 1 January to 31 March 1991 . There are $T=2160$ observations in all. The top left panel is an initial segment of the series. The autocovariance estimate here shows strong periodicity. The power spectrum of (3.5) is particularly useful in making inferences concerning periodicities and developing predictors. An estimate of a flattened version (see Appendix) is given in the bottom display of Figure 6. Peaks are seen to stand out. The presence of periodic components in tidal series is basic and ascribed to the effects of the moon and the sun. A pertinent model is provided by

$$
Y(t)=\mu+\sum_{k=1}^{K} \rho_{k} \cos \left(\omega_{k} t+\phi_{k}\right)+\epsilon(t)
$$

with $\epsilon$ a stationary noise series with smooth spectrum $f_{\epsilon \epsilon}$. If the $\phi_{k}$ are uniform and independent, the power spectrum of $Y$ is

$$
f_{Y Y}(\lambda)=\sum_{k} \frac{\rho_{k}^{2}}{4}\left\{\delta\left(\lambda-\omega_{k}\right)+\delta\left(\lambda+\omega_{k}\right)\right\}+f_{\epsilon \epsilon}(\lambda) .
$$

If $K=3$ in (3.9) and $\omega_{3}=\omega_{1}+\omega_{2}, \phi_{3}=\phi_{1}+\phi_{2}$ where $\phi_{1}, \phi_{2}$ are independent uniform, then the bispectrum has a term

$$
\frac{1}{8} \rho_{1} \rho_{2} \rho_{3} \delta\left(\lambda-\omega_{1}\right) \delta\left(\mu-\omega_{2}\right)
$$

for example.

Wood (1978) lists various estimates of tidal frequencies. The model (3.9) was fitted to the Saint John data by least squares, employing the $K=26$ frequencies of the final 
Vol. 22, No. 2

Saint John tides

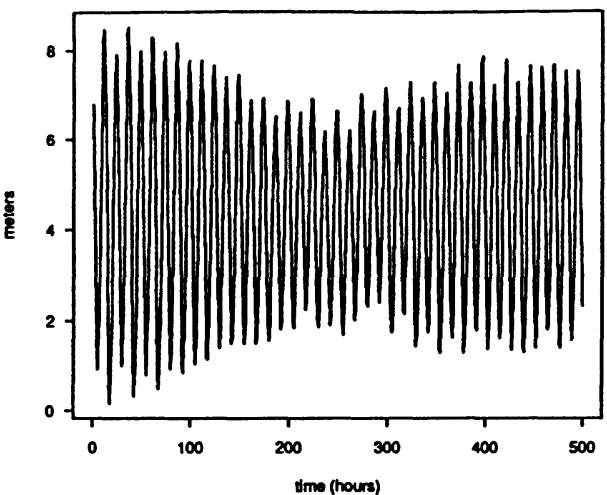

Autocovariance estimate

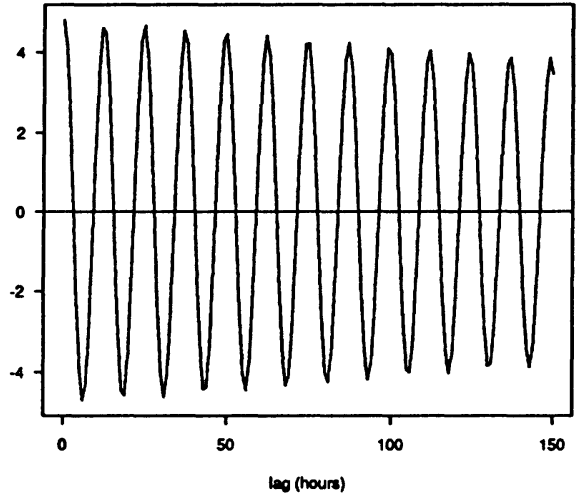

Periodogram

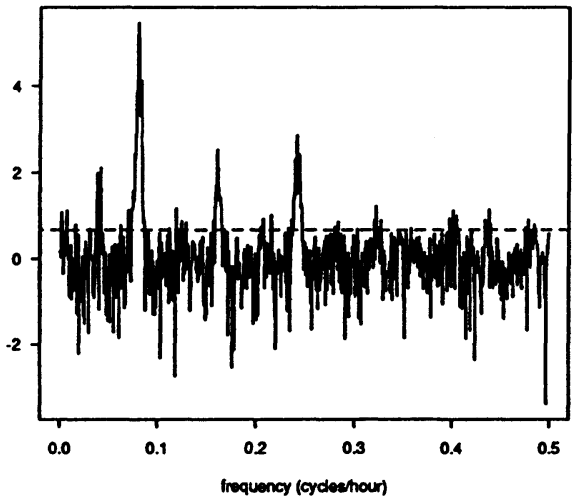

Residuals (26 components)

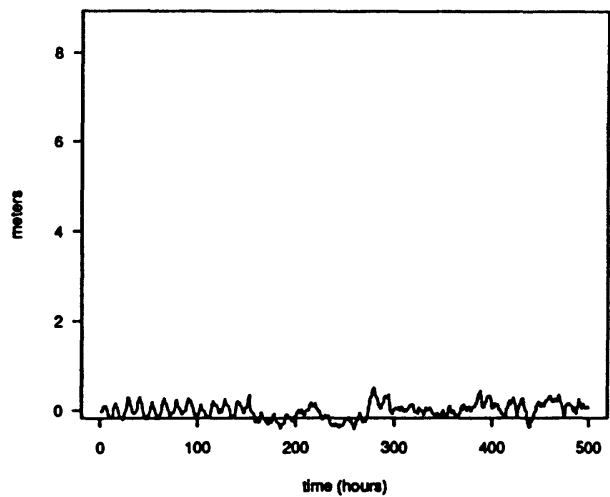

Autocovariance estimate

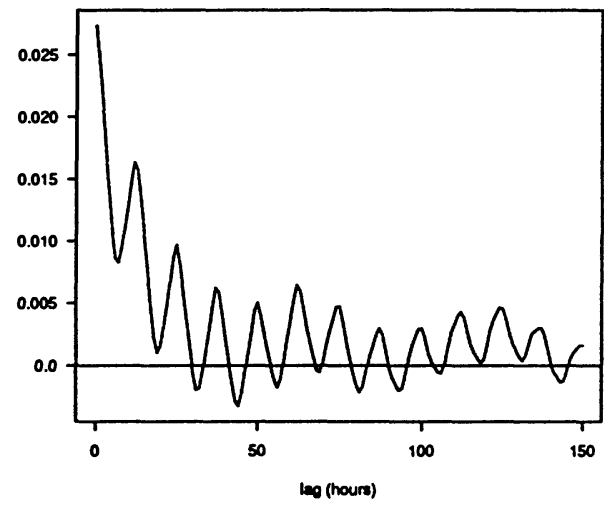

Periodogram

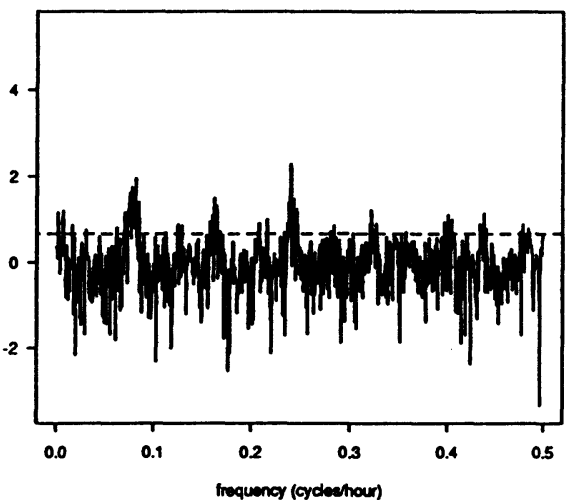

FIGURE 6: Saint John tides and residuals. The first column presents statistics for the tidal series; the second, statistics for the residuals of a least squares fit to the series. The top displays are initial sections of the series themselves. The middle row provides estimated autocovariance functions. The bottom row provides an estimate of the spectrum with the continuous component flattened. The dashed line is an approximate upper $95 \%$ confidence level above an estimate of the flattened noise spectrum. 
column of Figure 43 in Wood (1978). The right-hand column of Figure 6 graphs the results. The residuals are much smaller than the original series values. (Their standard error is $0.165 \mathrm{~m}$. The original standard error was $2.192 \mathrm{~m}$.) The autocovariance estimate of the residuals and a flattened power spectrum are also given. Some things remain to be accounted for: there remain clear peaks with structure about them, suggesting the possibility of time lags and nonlinearities.

In non-Gaussian circumstances and situations where a basic process has been transformed in a nonlinear fashion, the third-order cumulant function $c_{Y Y Y}(u, v)$ and bispectrum $f_{Y Y Y}(\lambda, \mu)$ of (3.8) and (3.6) are of importance. For example, squaring $\rho_{1} \cos \left(\omega_{1} t+\right.$ $\left.\phi_{1}\right)+\rho_{2} \cos \left(\omega_{2} t+\phi_{2}\right)$ of (3.9) with $K=2$ leads to, amongst others, a further term $\rho_{3} \cos \left(\omega_{3} t+\phi_{3}\right)$ with $\omega_{3}=\omega_{1}+\omega_{2}$ and $\phi_{3}=\phi_{1}+\phi_{2}$ and the bispectrum term (3.11).

Figure 7 (top) presents estimates of the third moment function (3.8) for the original series and for the residuals from the least-squares fit. Positive contours are graphed with a solid line, negative with a dashed line. The third-order cumulant estimate of the original data suggests periodicity and asymmetry. The structure in the case of the residuals is not apparent. The bottom displays of the figure provides estimates of

$$
\min \left\{\frac{\rho_{1}^{2}}{f_{\epsilon \epsilon}\left(\omega_{1}\right)}, \frac{\rho_{2}^{2}}{f_{\epsilon \epsilon}\left(\omega_{2}\right)}, \frac{\rho_{3}^{2}}{f_{\epsilon \epsilon}\left(\omega_{3}\right)}\right\}
$$

as a function of $\left(\omega_{1}, \omega_{2}\right)$, with $\omega_{3}=\omega_{1}+\omega_{2}$. This parameter is meant to examine the null hypothesis that at least one of the harmonic components at frequencies $\omega_{1}, \omega_{2}, \omega_{1}+\omega_{2}$ is absent; see Brillinger (1980). Further details are in the Appendix. Graphed are the values significant at the $1 \%$ level. There is clear structure present in the original series, and much of the structure remains in the residuals. There are strong suggestions of nonlinear interactions.

Cartwright (1969) discusses the generation of nonlinear interactions in tidal series. Marone and de Mesquita (1993) are concerned with estimating the bispectrum with lower-order information removed.

\subsection{The Point-Process Case.}

Suppose that the point process $N$ is described via times $\tau_{j}, j=0, \pm 1, \pm 2, \ldots$. A stepfunction description is provided by $N(t)=\#\left\{\tau_{j} \mid 0<\tau_{j} \leq t\right\}$. There are other useful representations for a point process. A representation that suggests immediate extensions of corresponding time-series procedures is

$$
Y(t)=\frac{d N(t)}{d t}=\sum_{j} \delta\left(t-\tau_{j}\right)
$$

with $d N(t) / d t$ a symbolic derivative of the process. This is an extension of the line plot of Figure 2 with the lines now having infinite height. From the representation (3.13) one sees, for example, that a linear filtering is given by

$$
\int a(t-u) Y(u) d u=\sum_{j} a\left(t-\tau_{j}\right)
$$

with $a(\cdot)$ the impulse response of the filter. Similarly the empirical Fourier transform is given by

$$
\int_{0}^{T} e^{-i \lambda t} Y(u) d u=\sum_{0 \leq \tau_{j}<T} e^{-i \lambda \tau_{j}}
$$


Third cumulant tides

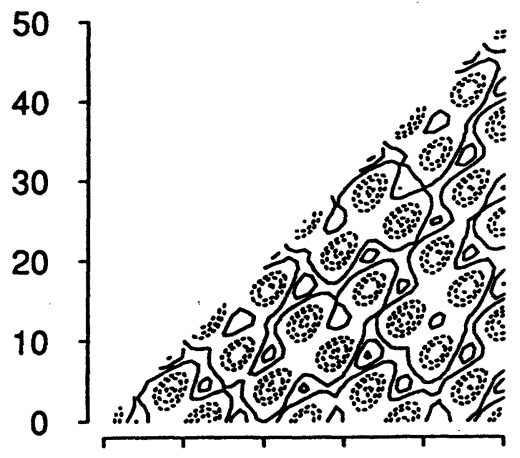

$\begin{array}{llllll}0 & 10 & 20 & 30 & 40 & 50\end{array}$

lag (hours)

Minimum periodogram tides

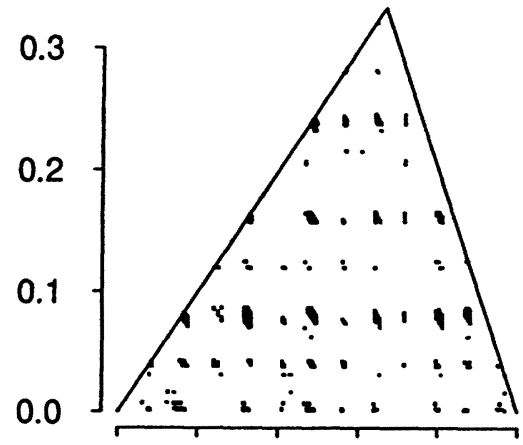

$\begin{array}{lll}0.0 & 0.2 & 0.4\end{array}$

frequency (cycles/hour)
Third cumulant residuals

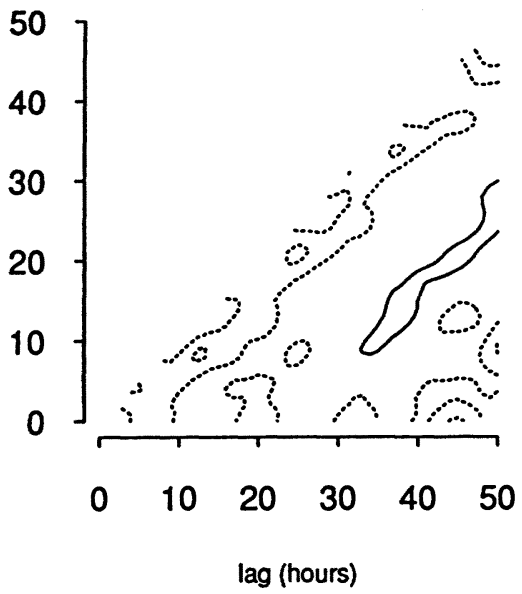

Minimum periodogram residuals

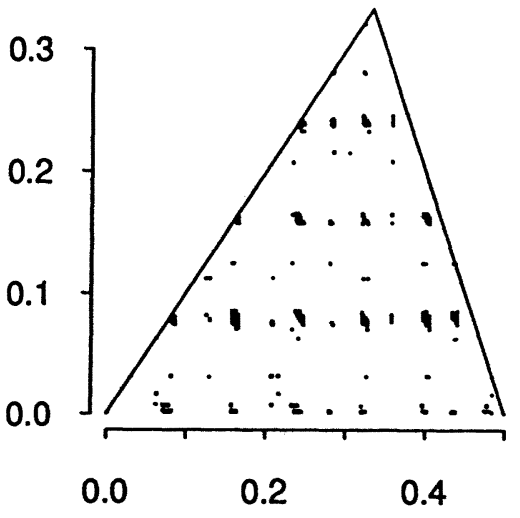

frequency (cycles/hour)

FIGURE 7: Top left is an estimate of the third-order cumulant function for the Saint John tidal data. Negative values are plotted as dashed lines. Top right provides the same for the residual series. The points significant at the $1 \%$ level of the statistic (A.5) are plotted for the two series.

It can also be convenient to consider a point process as a function of intervals, with $N(I)$ counting the points in the interval $I$. Then one has

$$
N(I)=\sum_{\tau_{j} \in I} 1=\int_{I} d N(t),
$$

and $N$ is seen to be a counting measure on the line.

A basic parameter of a stationary point process is the rate $p_{N}$, given by

$$
\operatorname{Prob}\{d N(t)=1\}=p_{N} d t=\mathcal{E}\{d N(t)\}
$$


for small $d t$. A second is the autointensity function $h_{N N}(u)$, given by

$$
\operatorname{Prob}\{d N(t+u)=1 \mid \text { point at } t\}=h_{N N}(u) d u, \quad u \neq 0 .
$$

The autointensity is a more primitive concept than the autocovariance, being based on a probability rather than the more technical idea of a moment. It is a direct measure of the chance of a further point occurring $u$ time units after an existing point.

Figure 8 presents an estimate of $h_{N N}(u)$ for each of two data sets. The top panels give illustrative segments of the data. The left-hand column corresponds to a sea-hare neuron firing regularly. The right column refers to the bursting neuron of Figure 3. The middle panels give the estimated autointensities. Complex periodic behaviour, which did not stand out in the firing-time display, is now apparent in the pacemaker case. The autointensity estimate in the bursting case has a broad peak at a lag of about 25 seconds, presumably corresponding to the spacings of the bursts. The bottom panels provide estimates of the power spectra $f_{N N}(\lambda)$, defined at (3.5), and bring out periodicities in an alternative fashion. The pacemaker firing does have a complex structure. The firing in the bursting case is seen to have a character suggesting harmonics.

The autocovariance density at lag $u, q_{N N}(u)$, of a stationary point process $N$ of rate $p_{N}$ is given by

$$
\operatorname{Cov}\{d N(t+u), d N(t)\}=\left[\delta(u) p_{N}+q_{N N}(u)\right] d t d u,
$$

while the third-order cumulant density is given by

$$
\begin{aligned}
\mathcal{E}\left\{\left[d N\left(t+u_{1}\right)-p_{N} d t\right]\left[d N\left(t+u_{2}\right)-p_{N} d t\right][d N(t)-\right. & \left.\left.p_{N} d t\right]\right\} \\
& =q_{N N N}\left(u_{1}, u_{2}\right) d t d u_{1} d u_{2}
\end{aligned}
$$

for $u_{1}, u_{2}, 0$ distinct.

Estimates of (3.15) for the pacemaker and bursting cases are given in Figure 9, top row. (Details of the construction of the estimates are found in the Appendix.) Positive contours are graphed with a solid line, negative ones with a dashed line. The periodic behaviors of Figure 8 show themselves in an alternative form. The bottom row of Figure 9 gives an estimate of the quantity (3.12), specifically (A.6) below, graphing points significant at the $1 \%$ level. There is a cluster at $(1.40,0.95)$ in the pacemaker case that might not have been suspected. The sum frequency, 2.35 , is apparent in the periodogram. The burst statistic likewise shows interesting structure.

\subsection{Hybrid Cases.}

Consider a marked point process case with real-valued marks. Realizations of the process have the form $\left\{\left(\tau_{j}, M_{j}\right), j=0, \pm 1, \pm 2, \ldots\right\}$. A representation for the process as a generalized ordinary time series is provided by

$$
Y(t)=\frac{d J(t)}{d t}=\sum_{j} M_{j} \delta\left(t-\tau_{j}\right)
$$

As a function of intervals, $J$ may be written

$$
J(I)=\sum_{\tau_{j} \in I} M_{j}
$$


L10 pacemaker
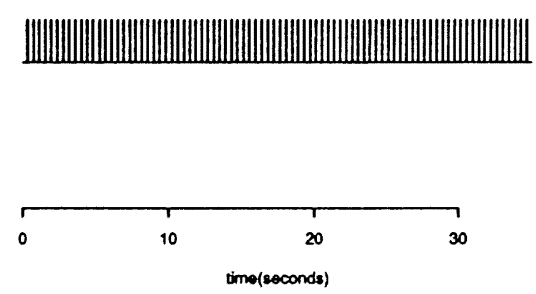

Autointensity estimate

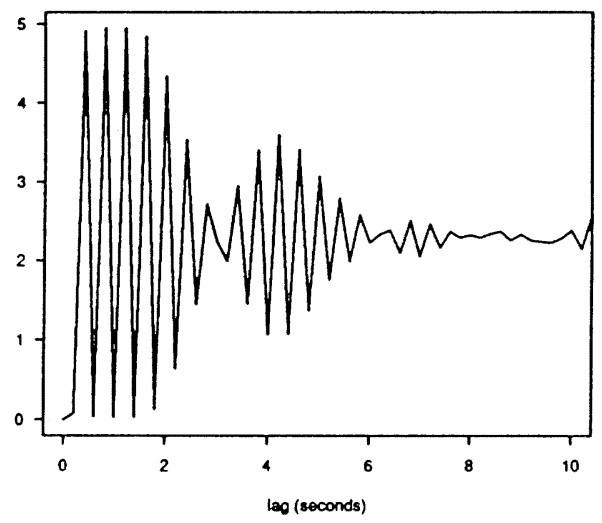

Periodogram

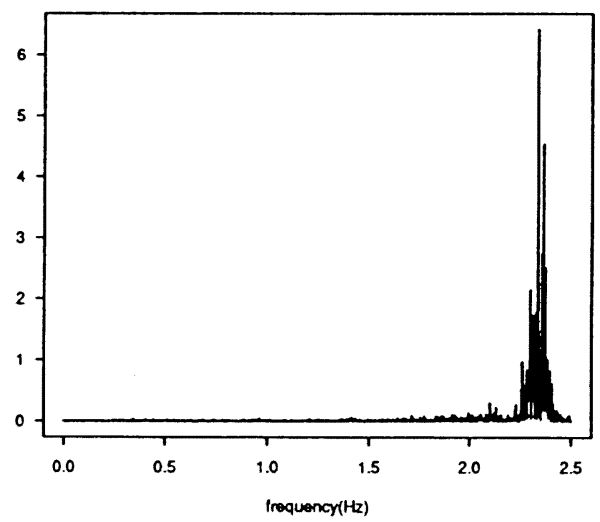

L10 bursting \& accelerando
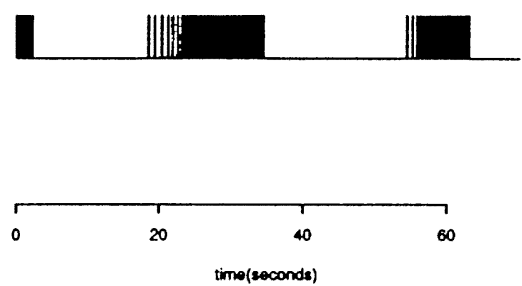

Autointensity estimate

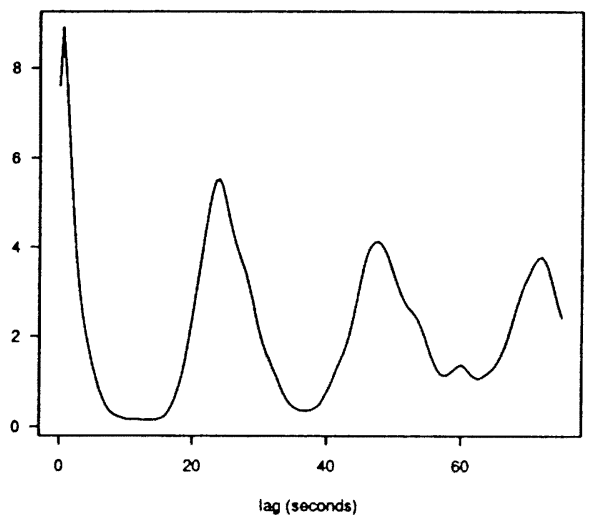

Periodogram

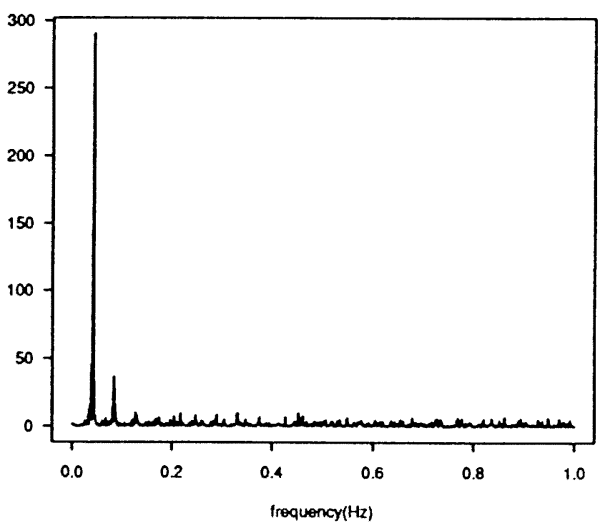

Figure 8: The left-hand panels give statistics for a neuron firing regularly, the right-hand for a neuron firing in bursts. Top are data sections. The middle displays are estimates of the autointensity [of (3.14)]. The bottom row provides the periodograms (A.5). 
Third cumulant pacemaker

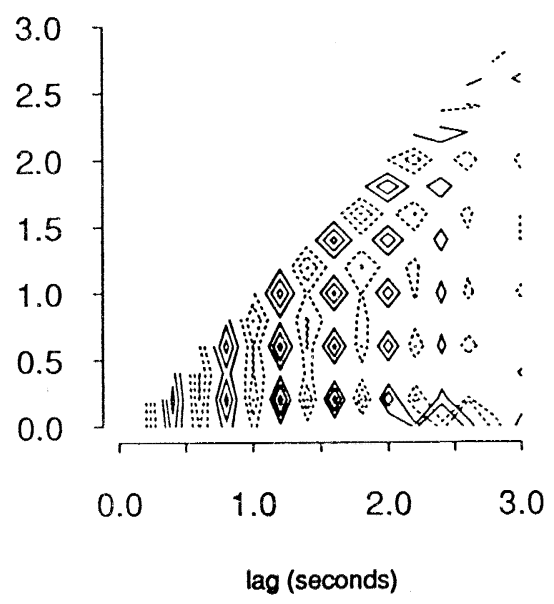

Minimum periodogram pacemaker

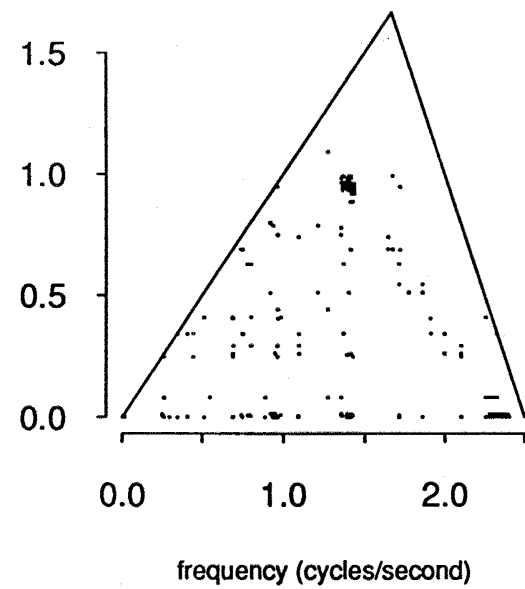

Third cumulant bursts

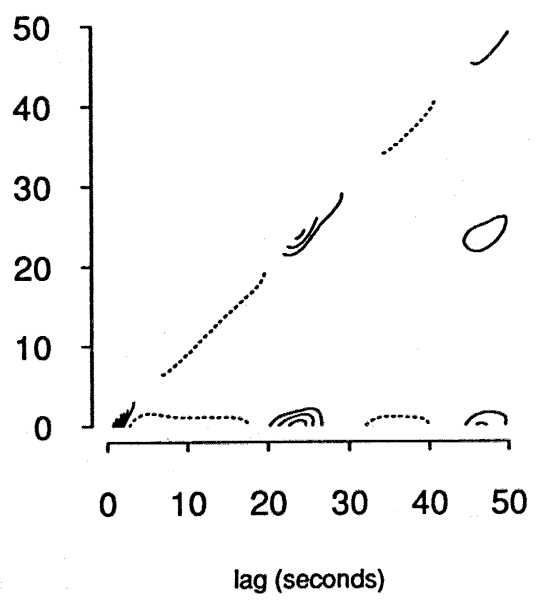

Minimum periodogram bursts

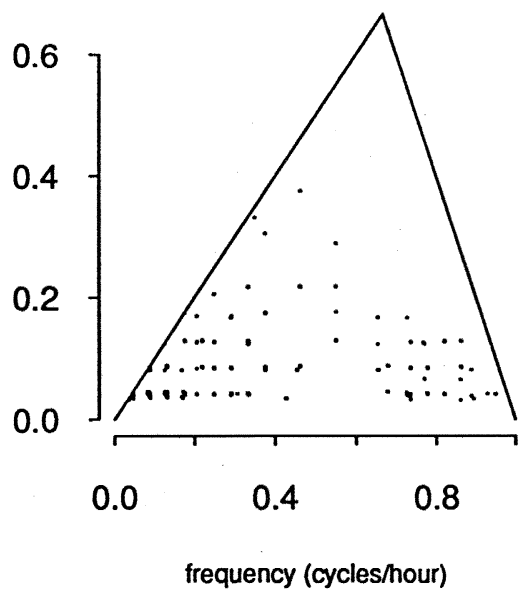

FigurE 9: The left-hand panels are the cumulant densities as estimated from (A.2). Negative contours are plotted as dashed. The bottom panels are the statistics (A.6) significant at the $1 \%$ level.

and is seen to correspond to a discrete measure on the line. The autocovariance density at lag $u, c_{J J}(u)$, of the process is given by

$$
\operatorname{Cov}\{d J(t+u), d J(t)\}=\left[\delta(u) c_{J}+c_{J J}(u)\right] d t d u
$$

with $\mathcal{E}\{d J(t)\}=c_{J} d t$.

A hybrid process is provided by a sampled ordinary time series, $\left\{Y\left(\tau_{j}\right)\right\}$. This can be represented via $d J(t)=Y(t) d N(t), N$ being the process of sampling times. This $J$ will have stationary increments when, for example, the processes $Y$ and $N$ are stationary and 
independent. A discrete time series corresponds to $\tau_{j}=j$. The spectral representation of $J$ involves

$$
d Z_{J}(\lambda)=\int d Z_{Y}(\lambda-\mu) d Z_{N}(\mu)
$$

a relationship from which expressions for various spectra may be obtained.

Figure 10 presents the initial stretch of the California earthquake data and of the corresponding point process of times. The second row left presents an estimate of the autocovariance $c_{J J}$. Below is an estimate of the power spectrum, $f_{J J}$. Approximate $95 \%$ marginal confidence intervals are indicated. No special structure is apparent.

An estimate of the third-order cumulant density is graphed in the top left of Figure 11. The bispectrum $f_{J J J}$ is given by

$$
\operatorname{cum}\left\{d Z_{J}(\lambda), d Z_{J}(\mu), d Z_{J}(v)\right\}=\delta(\lambda+\mu+v) f_{J J J}(\lambda, \mu) d \lambda d \mu d v,
$$

and the bicoherence by

$$
\frac{\left|f_{J J J}(\lambda, \mu)\right|^{2}}{f_{J J}(\lambda) f_{J J}(\mu) f_{J J}(\lambda+\mu)}
$$

Values significantly different from 0 at the $1 \%$ level are graphed in the bottom left of Figure 11. The results reflect the nonnormality of the process.

A question that arises when dealing with marked point processes is: are the series of marks, $\left\{M_{j}\right\}$, and the inherent point process, $N \equiv\left\{\tau_{j}\right\}$, independent of each other? This question may be addressed via a second-order moment analysis.

First some definitions pertinent to the bivariate case. The crosscovariance density at lag $u, c_{J N}(u)$, between the jump process $J$ and its inherent point process $N$ is given by

$$
\operatorname{Cov}\{d J(t+u), d N(t)\}=c_{J N}(u) d t d u
$$

for $u \neq 0$. Suppose that the marks $M_{j}=Y\left(\tau_{j}\right)$ correspond to sample values of a zero-mean stationary series $Y$. In the case that $Y$ and $N$ are independent, $c_{J N}$ will be identically 0 . So too will the cross-spectrum, $f_{J N}$, given by

$$
\mathcal{E}\left\{d Z_{J}(\lambda) d Z_{N}(\mu)\right\}=\delta(\lambda+\mu) f_{J N}(\lambda) d \lambda d \mu .
$$

Figure 10, middle right, graphs an estimate of $c_{J N}(u)$ for the California earthquake data. The values fluctuate about 0 . The sampling properties of an estimate of the coherence, $\left|R_{J N}(\lambda)\right|^{2}=\left|f_{J N}(\lambda)\right|^{2} / f_{J J}(\lambda) f_{N N}(\lambda)$, are simpler; hence this is the statistic employed to assess the independence. An estimate is graphed in Figure 10 bottom right. There is some evidence against independence: 21 points out of 128 exceed the $95 \%$ null point.

The third-order joint cumulant density may also be used to address the hypothesis of independence. It is given by

$$
\operatorname{cum}\{d J(t+u), d N(t+v), d N(t)\}=c_{J N N}(u, v) d t d u d v
$$

for $u, v, 0$ distinct. It will be 0 in the case of $Y$ independent of $N$. An estimate is given in Figure 11, top right. The crossbispectrum $f_{J N N}$ similarly will be 0 in the case of independence. An estimate based on the corresponding crossbicoherence is graphed in Figure 11. The points plotted are bifrequencies $(\lambda, \mu)$ where the bicoherence estimate is significantly different from 0 at the $1 \%$ level. There are many such points. A comparison 
California earthquakes 1932-1992

Marked point process

Point process
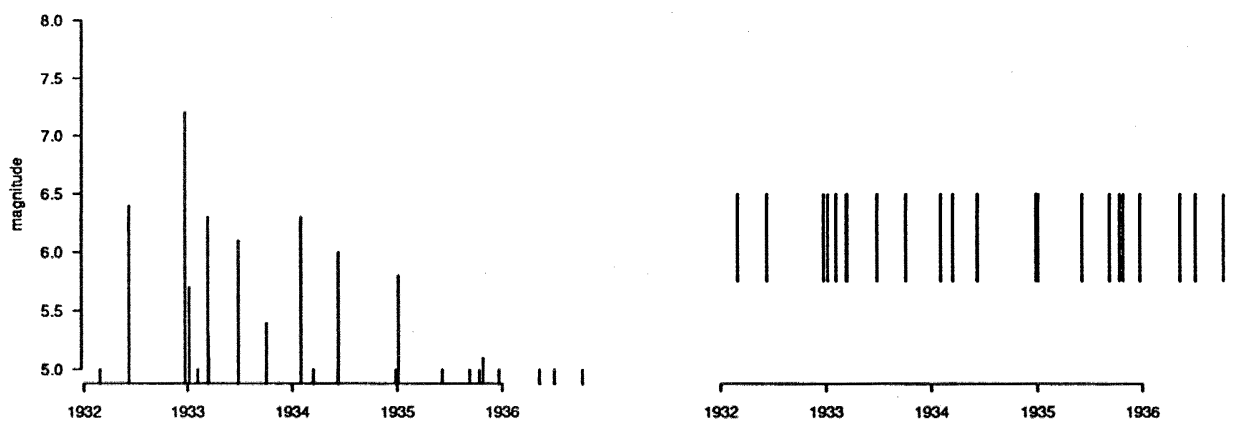

Autocovariance density

Crosscovariance density
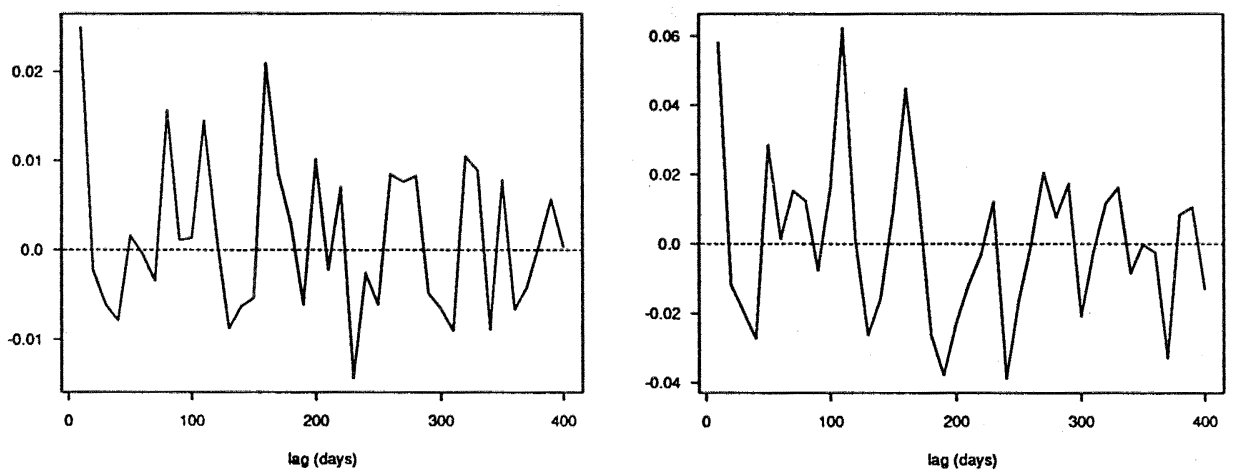

Power spectrum

Coherence
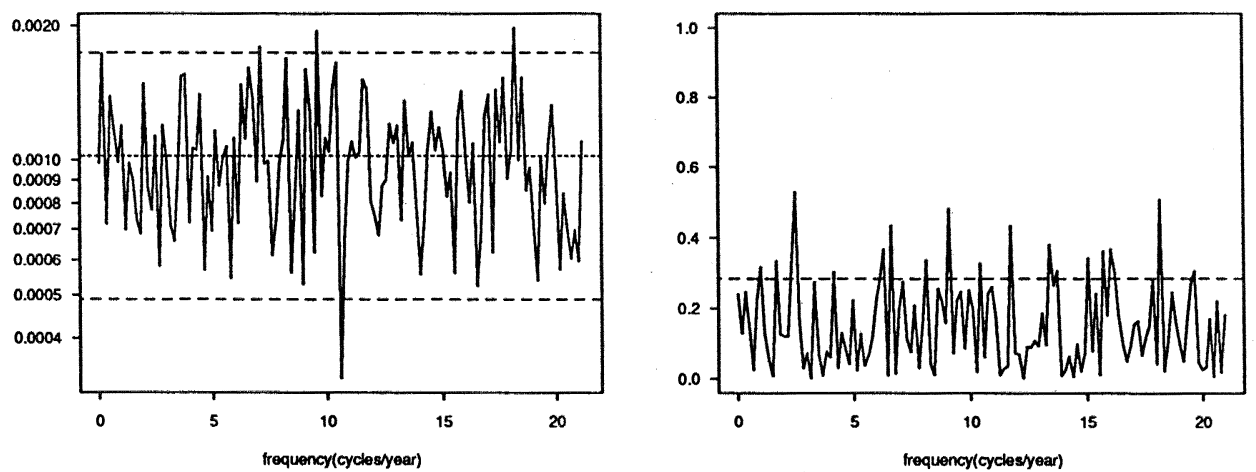

FIGURE 10: Analyses of the California earthquake data of Figure 4. The top displays are the magnitudes and times and just times (first 5 years). The bottom left is the powerspectrum estimate with an approximate $95 \%$ confidence interval set about the mean level. The bottom right is the estimated coherence with an upper $95 \%$ null level. 


\section{Third cumulant}

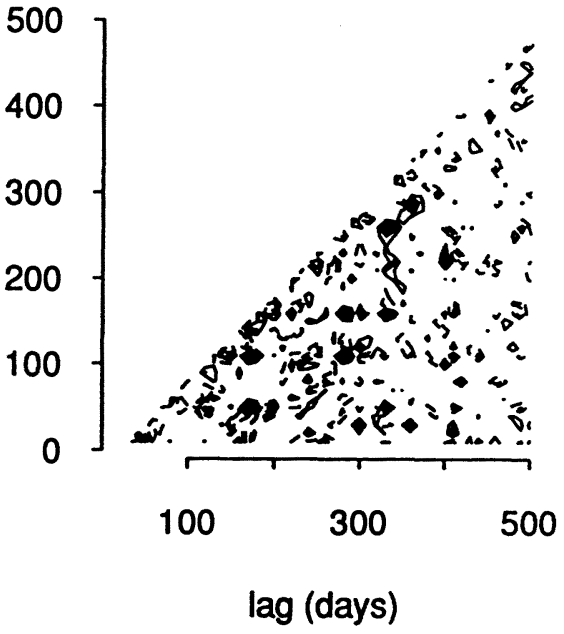

\section{Bicoherence}

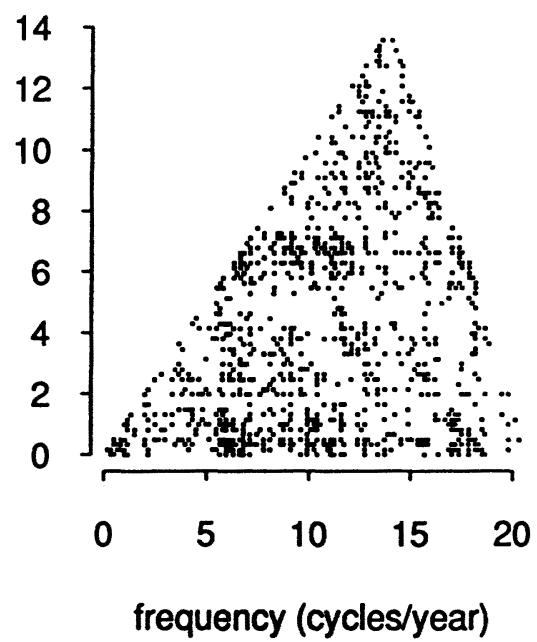

\section{Third joint cumulant}

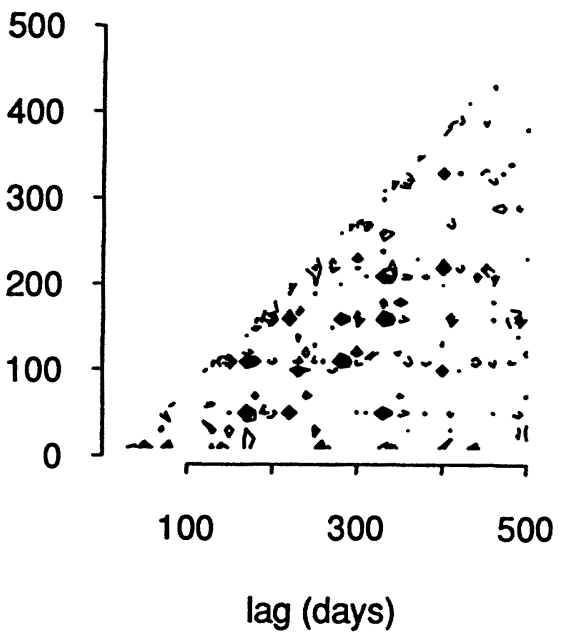

\section{Crossbicoherence}

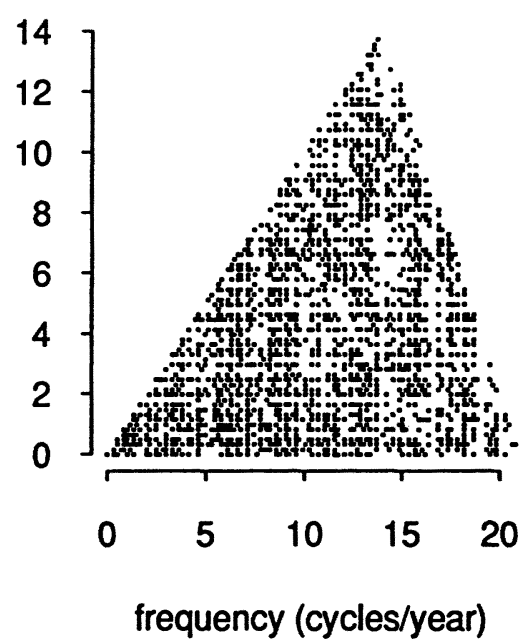

FIGURE 11: Estimates of third-order cumulant densities for the marked point process and point process. The bottom two graphs are points of the estimated bicoherence significant at the $1 \%$ level.

of the two bicoherences of Figure 11 shows many more significant points in the JNN case. This goes along with the process $(J, N, N)$ being more nonnormal.

Vere-Jones (1970) discussed point and marked point processes associated with earthquakes. The theory of point processes and marked point processes is presented in Daley and Vere-Jones (1988).

A question related to the present context is: assuming $Y$ and $N$ are independent, how 
does one estimate $c_{Y Y}$ and $f_{Y Y}$ ? One answer is given in Brillinger (1972). Some results of applying the technique are given in Moore et al. (1987).

\section{CONNECTIONS}

There are several methods for relating time series, point and marked point processes, and techniques. Advantages of employing these include: computing programs available for one type may be used with the others, models and theoretical results may be transferred, and generally further insight and understanding may be obtained.

A point process on the line may be studied via ordinary time-series methods through picking a small cell width $\delta$ and setting up the discrete time series

$$
Y(t)=N(t, t+\delta]
$$

for $t=0, \pm \delta, \pm 2 \delta, \ldots$ This 0 - 1 series may be fed to either moment- or likelihood-based techniques. For example, the second-order moments are connected via

$$
c_{Y Y}(u)=p_{N}(\delta-|u|)_{+}+q_{N N}(u) \delta^{2}
$$

for small $\delta$. The power spectrum of the discrete series (4.1) is given by

$$
f_{Y Y}(\lambda)=4\left(\sin \delta \frac{\lambda}{2}\right)^{2} \sum_{j=-\infty}^{\infty}\left(\lambda+\frac{2 \pi j}{\delta}\right)^{-2} f_{N N}\left(\lambda+\frac{2 \pi j}{\delta}\right)
$$

References include Vere-Jones and Davies (1966), Lewis (1970), Guttorp (1986), Guttorp and Thompson (1990).

The use of 0-1 series for point-process likelihoods occurs in Brillinger and Segundo (1979) and Berman and Turner (1992). When the model is correct, the likelihood approach may be anticipated to be the more efficient. However, the moment approach has the advantage of being broadly applicable and of having the same form for distinct types of processes. Indeed, if one moves to the frequency domain, the moment procedures are essentially the same for time series, point processes, and marked point processes.

A discrete time series $Y(t), t=0, \pm 1, \pm 2, \ldots$, may be set up as a planar point process via the correspondence $Y(t) \rightarrow(t, Y(t))$. A marked point process, with marks in $\mathbb{R}^{p}$, may similarly be considered a point process lying in $\mathbb{R}^{p+1}$ through the expedient of simply viewing $\left(\tau_{j}, M_{j}\right)$ as a point in $\mathbb{R}^{p+1}$. One reference is Karr (1976).

A jump process $J$ may be associated with a time series in continuous time through the correspondence

$$
Y(t)=\int a(t-u) d J(u)
$$

see Priestley (1963), Jowett and Vere-Jones (1972). The spectra are related by

$$
f_{Y \ldots Y}\left(\lambda_{1}, \ldots, \lambda_{k-1}\right)=A\left(\lambda_{1}\right) \cdots A\left(\lambda_{k-1}\right) \overline{A\left(\lambda_{1}+\cdots+\lambda_{k-1}\right)} f_{J \ldots J}\left(\lambda_{1}, \ldots, \lambda_{k-1}\right),
$$

on which estimates may be based. The $0-1$ time series above corresponds to $a(\cdot)$, a boxcar function of width $\delta$. Hence $A(\lambda)=2\left(\sin \frac{1}{2} \lambda \delta\right) / \lambda$, which is approximately $\delta$ for small $\delta$.

Parallel development of the time-series and point-process cases is provided in Brillinger (1978). 


\section{TWO CURRENT PROBLEMS}

\subsection{Cell Motility.}

Consider the movement of biological objects. Vale (1993) comments that "Understanding the mechanism by which biological motors work has been one of the great puzzles in biophysics for the last century ...”. The specific question that will be considered here is whether such motors produce stepwise movement with abrupt displacements, or whether the movement is diffusionlike.

In the experiments to be studied a motor (kinesin) was attached to a small bead. In the presence of adenosine triphosphate (ATP) the bead is transported along a microtubule. The location of the bead may be followed by means of a special microscope. At issue is whether the motion of the bead is smooth or jumpy.

Figure 12 shows data for two cases based on material extracted from the bovine brain. Details of the data collection are given in Malik et al. (1993). A microscope with nanometer precision and millisecond temporal resolution is employed in following the motion. The data as measured appear as a track in the plane (Figure 12, top). In case 1023 the tubule is rocking substantially from side to side, motion that may be due to the vibration of the microscope. In case 1639 the motion is more nearly confined to a line. The data were rotated to obtain motion in directions parallel and perpendicular to the general directions of movement. The parallel motions, $X$, are given in the second row of Figure 12. The figure also gives the histograms of the step sizes, $X(t+1)-X(t)$. There is no spike standing out in either, which would correspond to some common jump size.

A model that may be considered is the following. Let $X(t)$ be the position, as a function of time, of the bead along the line of movement. Suppose that the bead jumps a distance $\alpha$ at the times $\tau_{j}$ of a point process $N$ and that there is an associated noise process $E$. Here $\alpha$ corresponds to the distance between successive kinesin binding sites on the microtubule. It is about $8 \mathrm{~nm}$. Write

$$
X(t)=\mu+\alpha N(t)+E(t)
$$

This model involves both a time series and a point process, and it naturally leads to a process with stationary increments. In the case that $\alpha$ is small and the rate of the point process high, the particle will appear to be diffusing.

Supposing the processes $N$ and $E$ to have stationary increments, the expected value of $X(t)$ is $\mu+\alpha p_{N} t$, where $p_{N}$ is the rate of the process $N$. The velocity with which the particle is moving is $\alpha p_{N}$, and this will change with the experiment, but $\alpha$ will be constant across experiments. For $N$ and $E$ independent, the power spectrum of $X$ is

$$
f_{X X}(\lambda)=\alpha^{2} f_{N N}(\lambda)+f_{E E}(\lambda) \text {. }
$$

If $N$ is a renewal process, with interevent distribution having characteristic function $\phi$, then

$$
f_{N N}(\lambda)=\frac{p_{N}}{2 \pi} \frac{1-|\phi(\lambda)|^{2}}{|1-\phi(\lambda)|^{2}}
$$

[see (2.11.43) in Brillinger (1978)] and the rate of $N$ will be $p_{N}=1 / \mu$, where $\mu$ is the mean of the interevent distribution. In the case that the interjump time, $\tau_{j+1}-\tau_{j}$, is constant, and $v$ denotes the velocity of movement, the power spectrum is

$$
\alpha \sum_{j} \delta\left(\frac{\lambda}{v}-\frac{2 \pi}{\alpha} j\right),
$$

a function seen to depend on $\lambda / v$. 
2 D track, case 1023

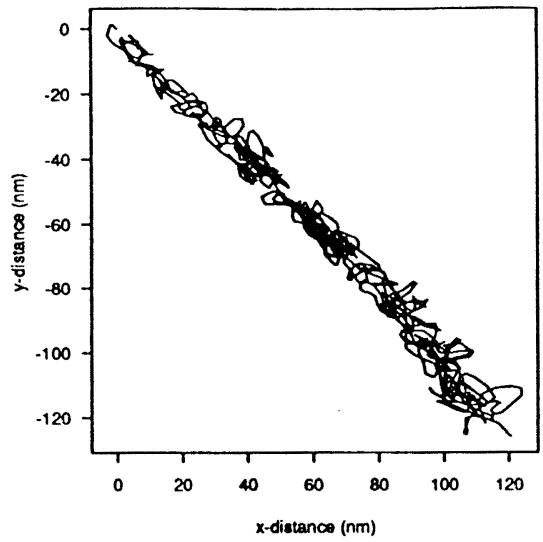

Parallel movement

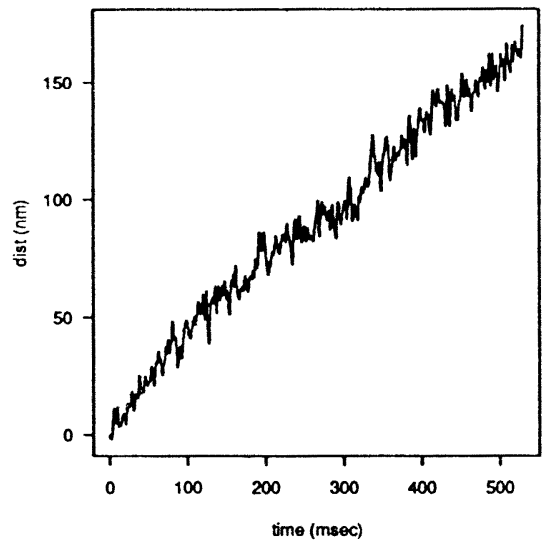

Steps

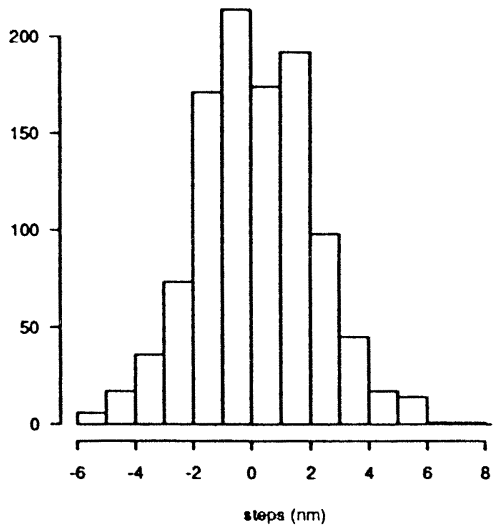

$2 \mathrm{D}$ track, case 1639

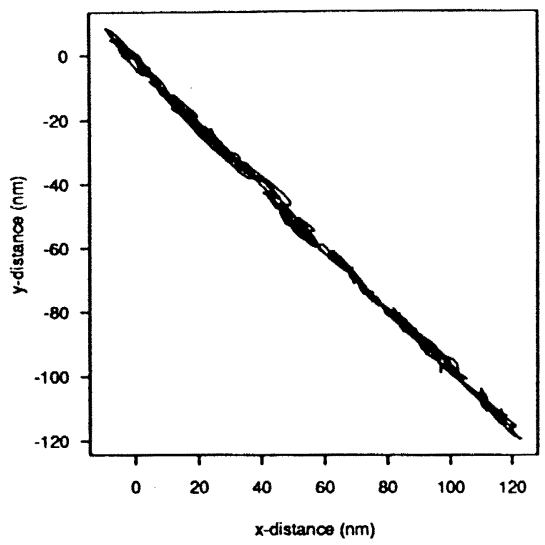

Parallel movement

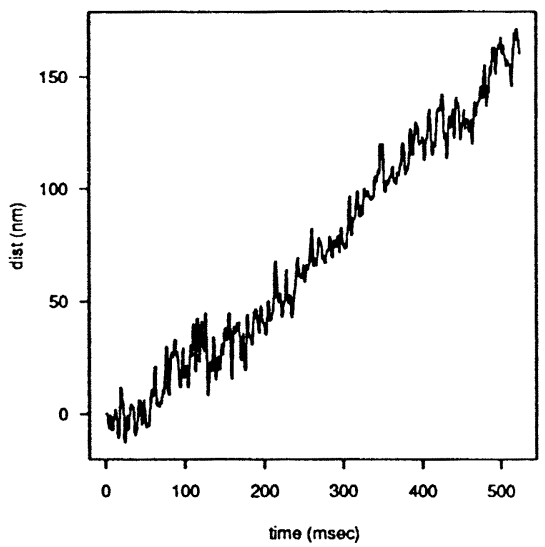

Steps

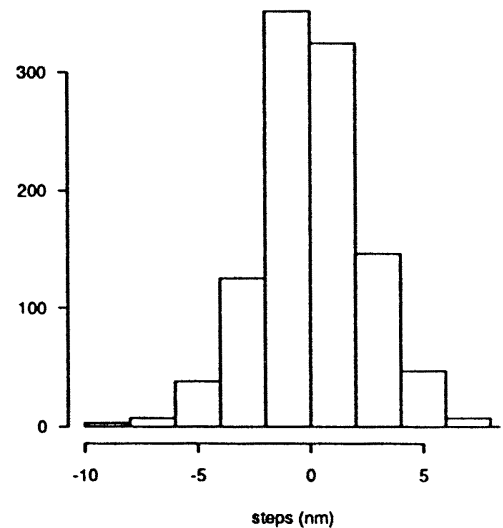

FIGURE 12: Microtubule movement in two experiments. The top graphs show the actual motion of the bead, starting from position $(0,0)$ in each case. The second row shows the motion, as a function of time, along the direction of motion, from the upper left to the bottom right corner of the top figures. The final displays are histograms of the individual steps. 
To address the issue of the size of $\alpha$, a procedure sensitive to periodic-type behaviour was employed. If the points occur in an approximately regular fashion, there will be peaks in the spectrum that will stand out. Suppose that the interevent density has the form $g(x / \theta) / \theta$ for a scale parameter $\theta$. The parameter $\theta$ will describe the velocity of the bead in a particular experiment: the larger $\theta$, the smaller the velocity. The spectrum $f_{N N}$ will be a function of $\theta \lambda$. So instead of plotting versus $\lambda$ one plots versus stepsize $=$ velocity $/ \lambda$. This allows the results of separate experiments to be combined. It is further noted that studies of the noise when the microtubule is not stimulated to move show the spectrum $f_{E E}$ to be smooth.

Figure 13 shows results of both simulations and data analyses. Renewal processes with interevent times gamma-distributed with coefficient of variation 0.2 were simulated. The top left display is an example of part of one of the step functions. Next, the step size was taken to be $8 \mathrm{~nm}$ and actual noise records added on. The top right provides the results of estimating the power spectrum from the simulations, flattening at the low-frequency end, transforming the abcissa to velocity/frequency, and averaging the results of 14 runs. A peak stands out at the $8 \mathrm{~nm}$ position, with a prob value of 0.009 . The procedure may now be considered validated in a sense. The bottom two displays correspond to applying the same steps to the parallel and perpendicular motions of 14 actual experiments. In these two cases the prob values of the largest peaks were 0.120 and 0.176 . There is no strong evidence for motion in jumps.

\subsection{Seal Diving.}

Marine ecologists are concerned with the navigation, foraging, and spatial-temporal use of the underwater habitat by marine mammals. Questions arise like: How are they moving? What are they doing? How are they interacting? The principal type of data that these scientists have worked with is depth as a function of time (such as the bottom display of Figure 15 below); hence for example they have been unable to estimate velocity, and there have been problems in identifying other quantities of interest. Recently three-dimensional data have started to become available.

Figure 14 displays the track of a ringed seal as recorded in the Barrow Strait, NWT. The ecology of these seals is described in Kelly (1988). The four figures correspond to viewpoints rotated 90 degrees from each other. This type of display is particularly effective when viewed spinning in real time.

The animal was in the wild, moving under ice from a breathing hole and returning. The seal had a sonic tag. Four hydrophones were placed at known locations, allowing the estimation of the seal's track; see for example Wartzok et al. (1992). There is measurement error in the estimates of position, and the time points are unequally spaced (hence one has marked-point-process data).

Figure 15 shows the individual $X(t), Y(t), Z(t)$ series. These traces bring out additional features of the data, for example that the seal stopped moving for a period. The $X$ and $Y$ traces show the track to be jumpy, and indeed this was apparent in Figure 14. These jumps can be due to measurement error. They are seen to occur at the same places in the $X$ and $Y$ traces. As a first step towards more complicated analyses an "improved" estimate of the track is needed. This is a problem of robust-resistant smoothing, with the twist that the dependent quantity is vector-valued; hence available procedures such as loess (see Becker et al. 1988) cannot be used efficiently. Figure 16 presents the results of univariate smoothing, via the function loess( ) of Chambers and Hastie (1992), but pooling the $X$ and $Y$ residuals together. One sees a plausible track for the animal, except 
Renewal step process

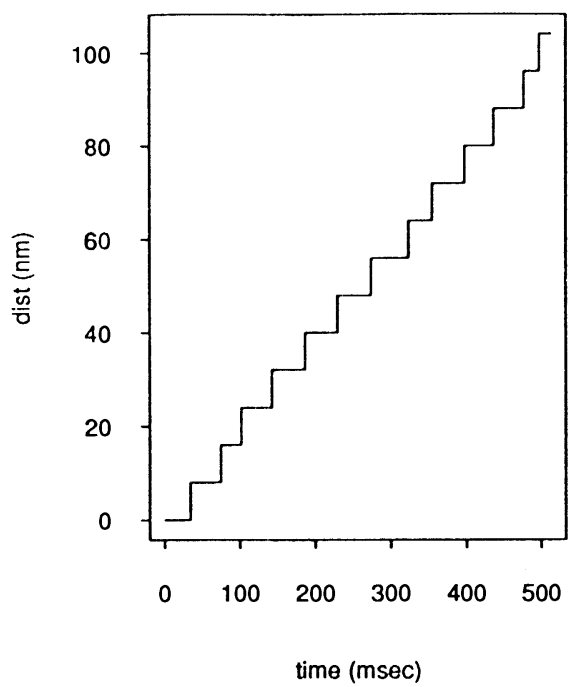

Average of parallel cases

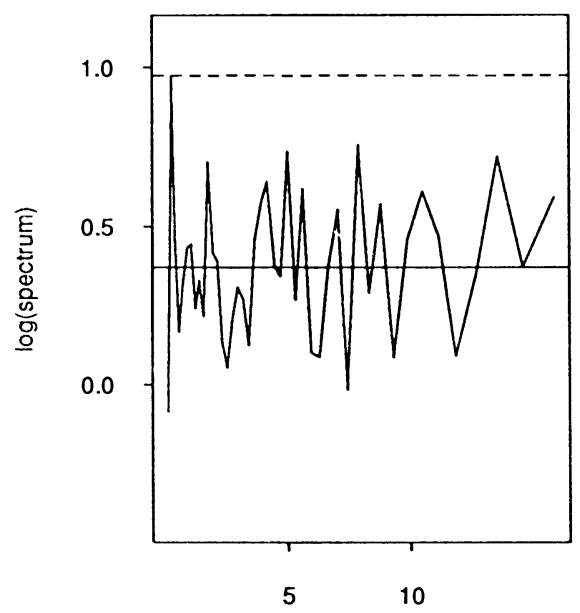

$\operatorname{step}(\mathrm{nm})$
Average $8 \mathrm{~nm}$ renewal cases

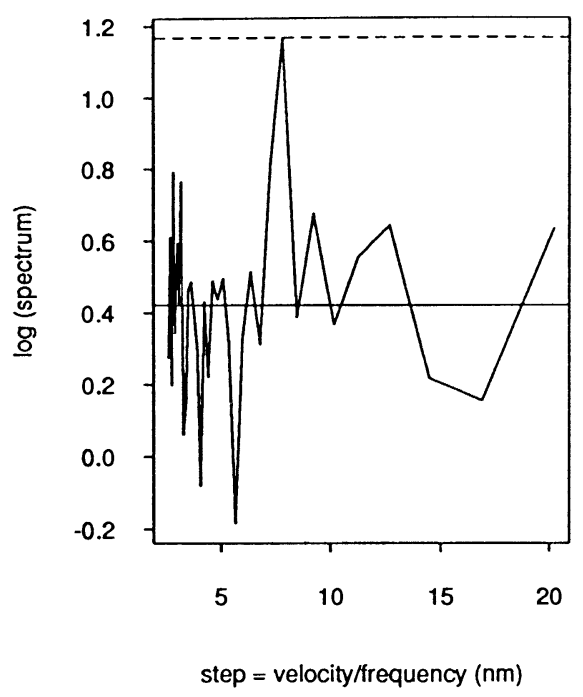

Average of perpendicular cases

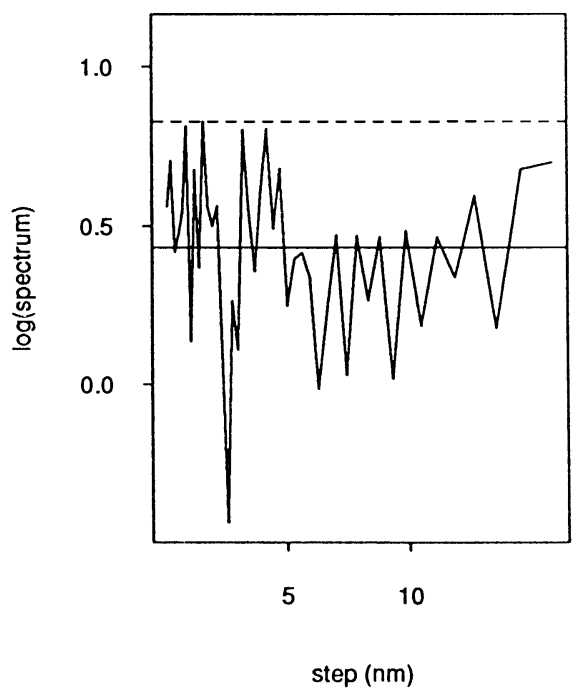

Figurf: 13: The top row refers to the results of simulations of a renewal process, the interevent distributions being gamma of coefficient of variation 0.2 and corresponding to an $\alpha$ of $8 \mathrm{~nm}$. The left display is a stretch from one of the simulations. On the right is the results of averaging the flattened, frequency-transformed spectra. The bottom displays are the results of applying the technique described to the parallel and perpendicular motions respectively. The solid horizontal line is at the average level. The dashed line is at the height of the highest ordinate. The significance levels in the three cases are $0.009,0.120,0.176$ respectively. 

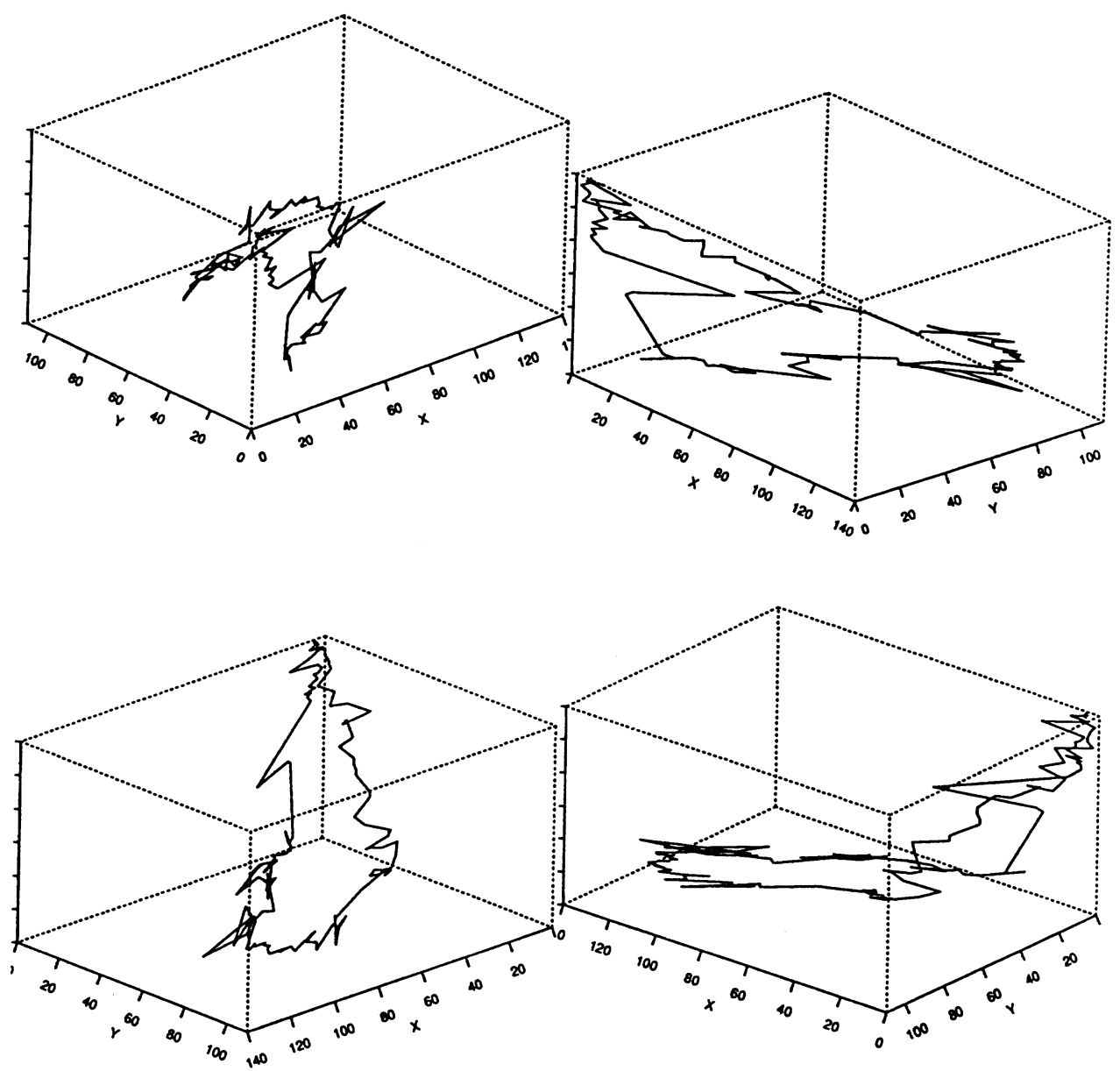

FIGURE 14: A track of a diving seal viewed from four perspectives rotated 90 degrees from each other. The distances are all in meters.

that now the resting period is not so apparent. The depth trace, $Z$, was not smoothed, because it appeared sufficiently regular already.

Another interesting aspect of these series is that they are tied down: the seal returns to its initial position. Also the surface and bottom form special barriers. These things must be taken note of in analytic models.

\section{DISCUSSION AND SUMMARY}

In her functioning, Nature appears to make use of each of time series, point processes, and marked point processes. This work has sought to bring out some parallel definitions and methods for these concepts. The models and techniques employed are mainly nonparametric and moment-based. Another aspect has been the illustration of both time-side and frequency-side analyses. Generally speaking the (approximate) sampling properties are simpler in the frequency domain.

Various displays were presented for each data type. In particular the tool of stacking has been highlighted as being of use in some circumstances.

A new statistic (A.6) has been employed in the study of discrete components in a bispectrum. The statistic has advantages over the biperiodogram, for the biperiodogram 

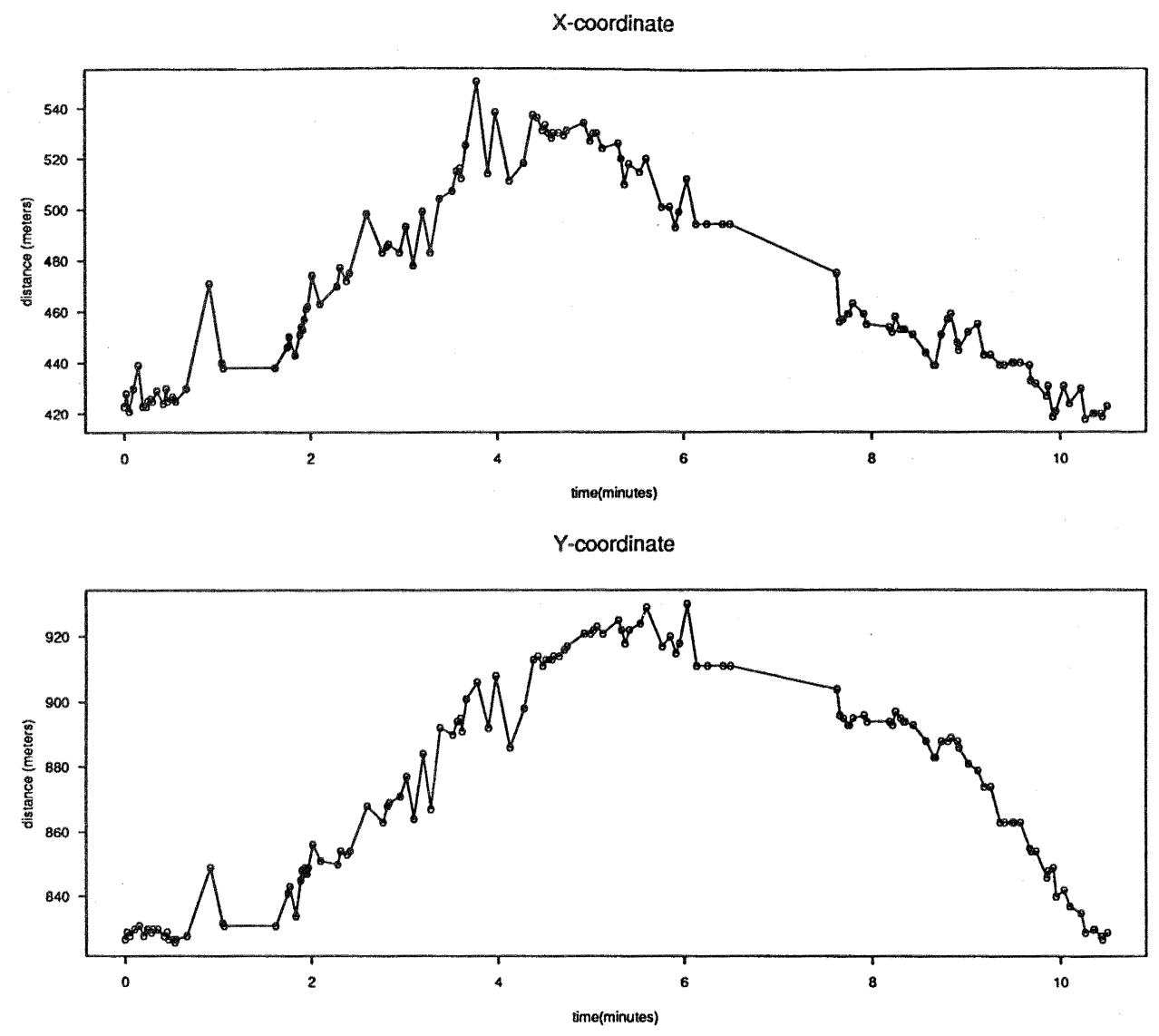

Z-coordinate

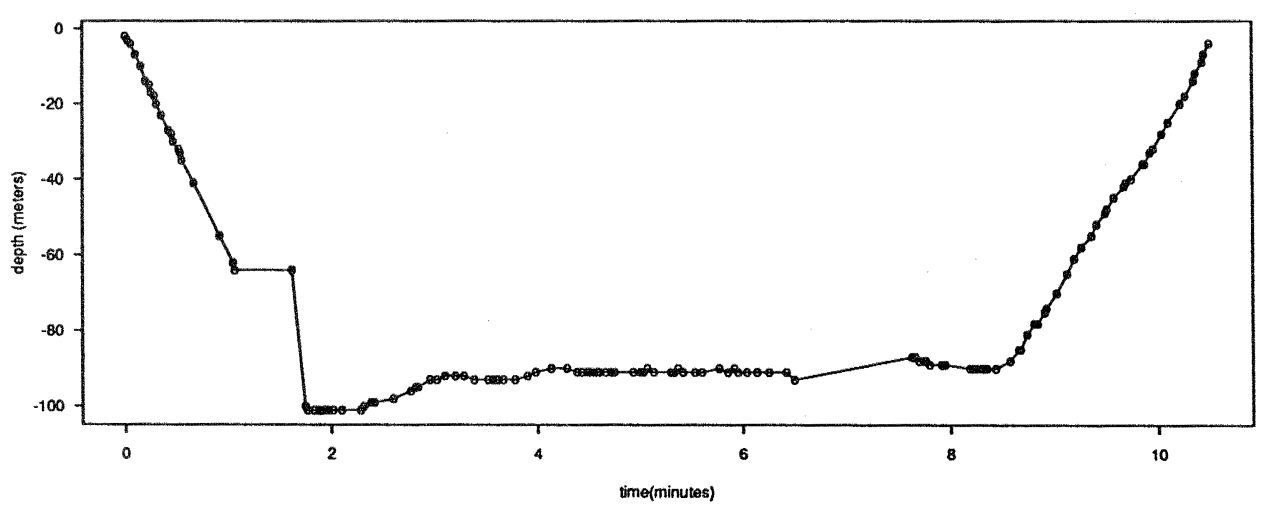

FigurE 15: Plots of $X, Y, Z$ versus time for the data of Figure 14. The points correspond to the times of measurement. 
Smoothed X-coordinate

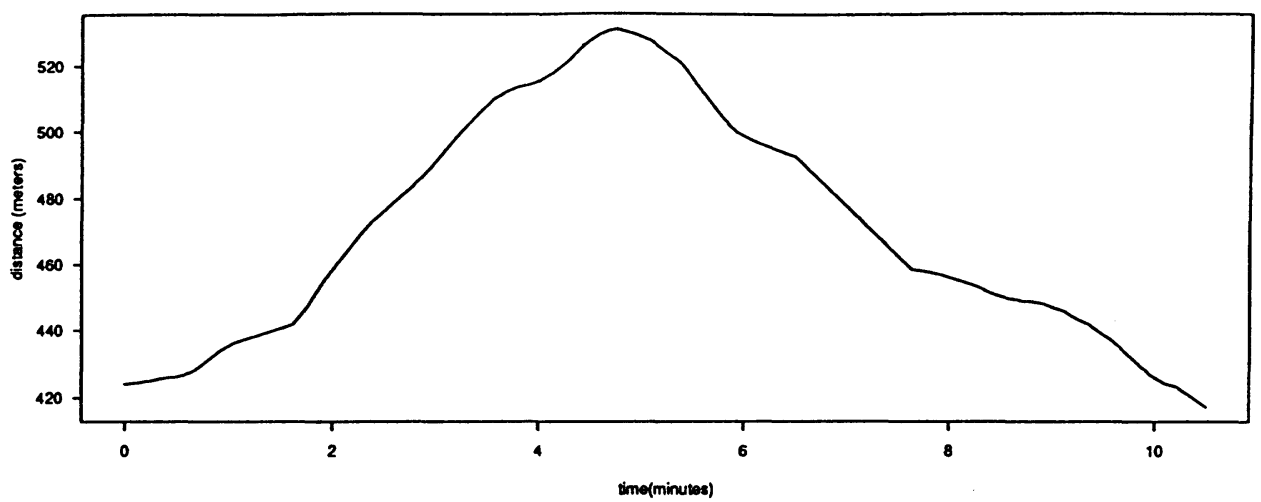

Smoothed Y-coordinate

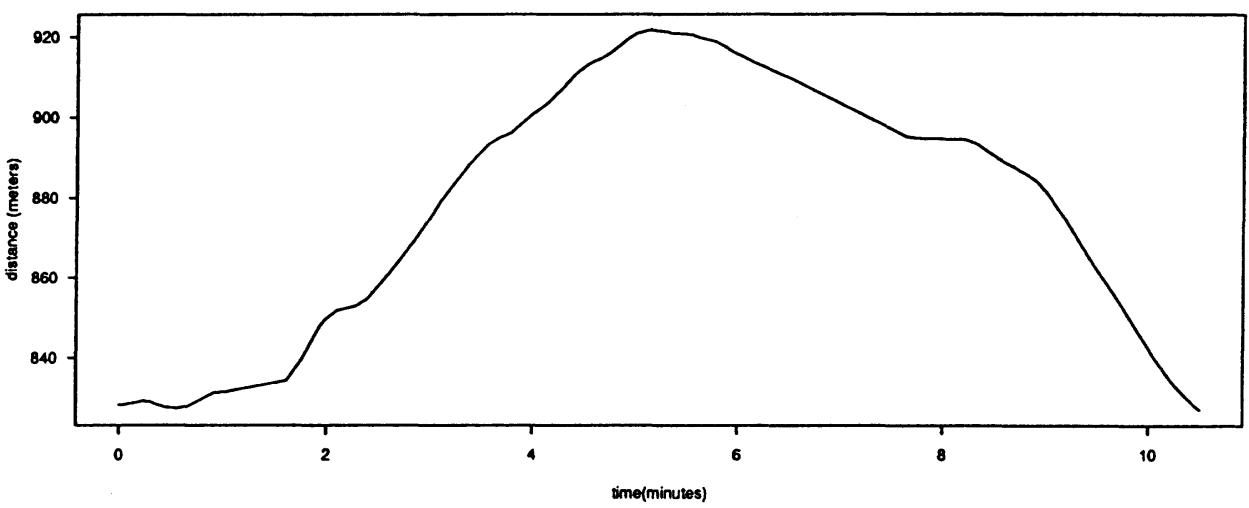

FIGURE 16: Robust-resistant smoothing of the $X$ and $Y$ traces of Figure 15.

will be large in amplitude when any of the frequency components involved is large. The statistic (A.6) standardizes for this.

Analyses were provided of data taken from five fields: oceanography, neurophysiology, seismology, biophysics, and ecology. In all studies it is good practice to ask: "What is the question?" Questions going along with the examples of this paper include:

(1) Tides. How to predict? The analyses presented were in part directed at understanding if an existing model was satisfactory.

(2) Nerve firings. How to describe? Description is needed because there are so many types of behaviour.

(3) Earthquake times and sizes. How to predict? One focus was on whether magnitudes were related to occurrence times; a second was on the presence of periodicities.

(4) Biological motors. Is the motion discrete or diffuse? One type of discrete motion, approximately regular jumping, was examined for via frequency-domain techniques.

(5) Seal tracks. How to estimate and display the motion? A spinning display proved effective, and robust-resistant smoothing looked promising.

\section{FUTURE DIRECTIONS}

There are a number of directions in which the work may be extended. The analyses employed have been based on second- and third-order moments principally. Extensions 
can be pursued to higher-order moments and to likelihood analyses. The existing techniques need to be extended to include covariates and to handle nonstationarity. The case of trends is discussed in Brillinger (1993). The marks of a marked point process may be ordinal, interval, nominal, ranks, or vector-valued, and corresponding special techniques need to be developed.

There are interesting analytic problems. For the estimates (A.1), (A.2), the question of how to choose the cellwidths $\delta$ and $b$ needs to be addressed. There has been research on the corresponding question in spectrum estimation, but not in product-density cases. The sampling properties of the flattened spectrum estimate [the statistic (A.6)] and of the argument-transformed estimate of Section 5.1 need to be studied in detail.

Suppose there are tracks of several particles. How is one to model and describe the way they are interacting? As in the case of Section 5.2, models may be needed to handle the fact that $(X(t), Y(t))$ may be tied down or bounded. As mentioned above, a multivariate version of loess needs to be developed. So too do other improved estimates of tracks based on irregularly observed data.

Stacking appears a powerful tool worthy of an in-depth study.

The cases of vector-valued processes and series need to be developed. So too do the spatial, marked spatial, and spatial-temporal cases. Some references to the spatial case include Hanisch and Stoyan (1979), Isham (1987), Ogata and Katsura (1988), Stoyan (1984).

\section{APPENDIX}

This section provides some details of the estimates and computations. Given data, $X(t)$, $0 \leq t<T$, general estimates of the $f_{X \ldots X}$ and $M_{X \ldots X}$ are indicated in Brillinger (1972) for processes with stationary increments.

If $\bar{Y}$ denotes the mean of the data $Y(t), t=0, \ldots, T-1$, of a discrete time series, then an estimate of the autocovariance function is

$$
c_{Y Y}^{T}(u)=\frac{1}{T} \sum_{t=0}^{T-|u|}\{Y(t+u)-\bar{Y}\}\{Y(t)-\bar{Y}\},
$$

and of the third cumulant function is

$$
c_{Y Y Y}^{T}(u, v)=\frac{1}{T} \sum_{0 \leq t, t+u, t+v \leq T-1}\{Y(t+u)-\bar{Y}\}\{Y(t+v)-\bar{Y}\}\{Y(t)-\bar{Y}\} .
$$

These appear in Figures 6 and 7.

An estimate of the rate of a point process $N$ is $p_{N}^{T}=N(T) / T$, while an estimate of the autointensity is

$$
h_{N N}^{T}(u)=\frac{\#\left\{\left|\tau_{j}-\tau_{k}-u\right|<b, j \neq k\right\}}{2 b N(T)} .
$$

The estimate (A.1) was introduced in Griffith and Horn (1963) and considered in Cox (1965). It appears in Figure 8.

Following the discussion of Section 4 , an estimate of the third-order cumulant density at $u, v, 0$ distinct is given by

$$
q_{N N N}^{T}(u, v)=c_{Y Y Y}^{T}(u, v) / \delta^{3},
$$

where $Y$ is the corresponding $0-1$ time series based on cells of small width $\delta$. This appears in Figure 9. 
For a marked point process, given a binwidth $b$, one can consider the statistic

$$
\sum_{\left|\tau_{j}-\tau_{k}-u\right|<b} M_{j} M_{k}=\iint_{|t-s-u|<b} d J(t) d J(s)
$$

(with $j \neq k$ and $t \neq s$ ) in analogy with (A.1). One bases an estimate of $\operatorname{Cov}\{d J(t+$ $u), d N(t)\}$ on

$$
\sum_{\left|\tau_{j}-\tau_{k}-u\right|<b} M_{j}=\iint_{|t-s-u|<b} d J(t) d N(s)
$$

These appear in Figure 10, having adjusted the marks to mean 0.

In estimating frequency-domain parameters it can be convenient to work with the empirical Fourier transform

$$
d_{X}^{T}(\lambda)=\int_{0}^{T} e^{-i \lambda t} d X(t)
$$

In the cases of a discrete time series, a point process, and a marked point process this becomes

$$
\sum_{t} Y(t) e^{-i t \lambda}, \quad \sum_{j} e^{-i \tau i}, \quad \sum_{j} M_{j} e^{-i \tau i} \lambda
$$

respectively. These satisfy central limit theorems in various circumstances, allowing approximate distributions of derived statistics to be set down.

A crude estimate of the power spectrum is provided by the periodogram

$$
I^{T}(\lambda)=\frac{1}{2 \pi T}\left|d^{T}(\lambda)\right|^{2} .
$$

This appears in Figure 8.

The spectrum (3.10) shows lines superposed on a (smooth) curve. To make the lines stand out more, the data have been tapered prior to Fourier transforming and the curve is flattened. The flattening was done by applying a resistant heavy smoother to the log periodogram values to obtain an estimate of the spectrum, which is then divided out. In a related context Tukey (1963) suggests dividing the periodogram by the result of a repeated running median, and in a testing situation Chiu (1989) suggests dividing by trimmed means of periodograms.

A cross-spectral estimate $f_{J N}^{T}$ may be computed by breaking a data set of length $T$ into $L$ segments of length $V$, computing the crossperiodogram $(2 \pi V)^{-1} d_{J}^{V} \overline{d_{N}^{V}}$ for each, and averaging. The coherence may then be estimated by $\left|f_{J N}^{T}\right|^{2} f_{J J}^{T} f_{N N}^{T}$. Likewise, a bispectrum estimate may be obtained by averaging the biperiodograms

$$
\frac{1}{(2 \pi)^{2} V} d^{V}(\lambda) d^{V}(v) \overline{d^{V}(\lambda+\mu)} \text {. }
$$

The bicoherence may be estimated via

$$
\left|f_{J J J}^{T}\right|^{2} / f_{J J}^{T} f_{J J}^{T} f_{J J}^{T}
$$

Its distribution, in the case that the population value $f_{J J J}$ is 0 , is exponential with mean $V / 2 \pi L$. See Huber et al. (1971). 
In the case of a line in the bispectrum at $(\lambda, \mu)$, it can be more useful to consider the statistic

$$
\min \left\{\frac{\left|I^{T}(\lambda)\right|^{2}}{f^{T}(\lambda)}, \frac{\left|I^{T}(\mu)\right|^{2}}{f^{T}(\mu)}, \frac{\left|I^{T}(\lambda+\mu)\right|^{2}}{f^{T}(\lambda+\mu)}\right\}
$$

with $I^{T}$ the periodogram and $f^{T}$ a heavily smoothed resistant estimate of the power spectrum. The large-sample distribution of (A.6) under the null hypothesis that at least one of $\rho_{1}, \rho_{2}, \rho_{3}=0$ is that of

$$
\min \left\{e_{1}, e_{2}, e_{3}\right\}, \min \left\{e_{1}, e_{2}\right\}, e_{1},
$$

where the $e$ 's are independent exponentials depending on whether all $\rho$ 's, two, or just one are hypothesized. The critical value employed is based on the last, corresponding to the "worst" case, i.e., that closest to the alternative.

\section{ACKNOWLEDGEMENTS}

The author is grateful to Brendan Kelly of the University of Alaska, Fairbanks, for providing the ringed-seal data; to Fady Malik and Ron Vale of the University of California, San Francisco, for providing the microtubule data; to Pepe Segundo of the University of California, Los Angeles, for providing the sea-hare data; and to Keith Thompson of Dalhousie University for the Saint John data. These scientists also provided important ideas and discussion. The plots and many of the analyses were prepared in S; see Becker, Chambers, and Wilks (1988).

Je veux dire merci beaucoup à Niklaus Hengartner pour l'assistance fratemelle avec les acétates. I also thank the referees for a very careful reading of the paper and helpful suggestions.

\section{REFERENCES}

Bartlett, M.S. (1967). The spectral analysis of line processes. Proc. Fifth Berkeley Symp. Math. Statist. Probab. Volume 3 (L.M. Le Cam, J. Neyman, and E.L. Scott, eds.). Univ. of California Press, Berkeley, 135-152.

Becker, R.A., Chambers, J.M., and Wilks, A.R. (1988). The New S Language. Wadsworth, Pacific Grove, Calif. Berman, M., and Turner, T.R. (1992). Approximating point process likelihoods using GLIM. Appl. Statist., 41, 31-38.

Brillinger, D.R. (1972). The spectral analysis of stationary interval functions. Proc. Sixth Berkeley Symp. Math. Statist. Probab. Volume 1 (L.M. Le Cam, J. Neyman, and E.L. Scott, eds.). Univ. of California Press, Berkeley, 483-513.

Brillinger, D.R. (1974). The asymptotic distribution of the Whittaker periodogram and a related chi-squared statistic for stationary processes. Biometrika, 61, 419-422.

Brillinger, D.R. (1978). Comparative aspects of the study of ordinary time series and of point process, Developments in Statistics. Volume 1 (P.R. Krishnaiah, ed.). Academic Press, New York, 33-133.

Brillinger, D.R. (1980). The comparison of least squares and third-order periodogram procedures in the estimation of bifrequency. J. Time Series Anal., 1, 95-102.

Brillinger, D.R. (1993). Trend analysis: Time series and point process problems. Proc. Internat. Conf. Stochastic Statist. Methods in Hydrology and Environmental Engineering. Univ. of Waterloo.

Brillinger, D.R., and Segundo, J.P. (1979). Empirical examination of the threshold model of neuron firing. Biol. Cybernet., 35, 215-220.

Bryant, H.L., Jr., Ruiz Marcos, A., and Segundo, J.P. (1973). Correlations of neuronal spike discharges produced by monosynaptic connections and common inputs. J. Neurophysiol., 36, 205-225.

Buys-Ballot, C.H.D. (1847). Les Changements Périodiques de Température. Utrecht.

Cartwright, D.E. (1969). A unified analysis of tides and surges round north and east Britain. Philos. Trans. Roy. Soc. London, 263, 1-55.

Chambers, J.M., and Hastie, T.J. (1992). Statistical Models in S. Wadsworth, Pacific Grove, Calif.

Chiu, S-T. (1989). Detecting periodic components in a white Gaussian time series. J. Royal Statist. Soc. Ser. $B, 51,249-259$.

Cleveland, W.S. (1985). The Elements of Graphing Data. Wadsworth, Pacific Grove, Calif.

Cox, D.R. (1965). On the estimation of the intensity function of a stationary point process. J. Roy. Statist. Soc. Ser. B, 27, 332-337.

Cox, D.R., and Lewis, P.A.W. (1966). The Statistical Analysis of Series of Events. Methuen, London. 
Daley, D.J., and Vere-Jones, D. (1988). An Introduction of the Theory of Point Processes. Springer-Verlag, New York.

Davison, C. (1928). The annual periodicity of earthquakes. Bull. Seismol. Soc. Amer., 18, 246-266.

Doob, J.L. (1953). Stochastic Processes. Wiley, New York.

Forrester, W.D. (1983). Canadian Tidal Manual. Government of Canada, Ottawa.

Griffith, J.S., and Horn, G. (1963). Functional coupling between cells in the visual cortex of the unrestrained cat. Nature, 199, 876, 893-895.

Guttorp, P. (1986). On binary time series obtained from continuous time point processes describing rainfall. Water Resources Res., 22, 897-904.

Guttorp, P., and Thompson, M.L. (1990). Nonparametric estimation of intensities for sampled counting processes. J. Roy. Statist. Soc. Ser. B, 52, 157-173.

Hanisch, K.-H., and Stoyan, D. (1979). Formulas for the second-order analysis of marked point processes. Math. Oper. Statist., 10, 555-560.

Huber, P.J., Kleiner, B., and Gasser, T. (1971). Statistical methods for investigating phase relations in stationary stochastic processes. IEEE Trans. Audio Electron., AU-19, 78-86.

Isham, V. (1987). Marked point processes and their correlations. Spatial Processes and Spatial Time Series Analysis (F. Droesbeke, ed.). Univ. Saint-Louis, Brussels, 63-75.

Jowett, J., and Vere-Jones, D. (1972). The prediction of stationary point processes. Stochastic Point Processes (P.A.W. Lewis, ed.). Wiley, New York, 405-435.

Karr, A.F. (1976). Two extreme value processes arising in hydrology. J. Appl. Probab., 13, 190-194.

Katsumura, K. (1985). On regional characteristics of seasonal variation of shallow earthquake activities in the world. D.Sc. Thesis, Kyoto University.

Kelly, B.P. (1988). Ringed seal. Selected Marine Mammals of Alaska (W. Lentfer, ed.). Marine Mammal Commission, Washington, 57-75.

Laplace, P.S. Marquis de (1825). Traité de Mécanique Céleste 5. Bachelier, Paris.

Lewis, P.A.W. (1970). Remarks on the theory, computation and application of the spectral analysis of series of events. J. Sound Vibration, 12, 353-375.

Malik, F., Brillinger, D.R., and Vale, R.D. (1993). High resolution tracking of microtubule motility driven by a single kinesin motor. Preprint, University of California.

Marone, E., and de Mesquita, A.R. (1993). On non-linear analysis of tidal observations. Continental Shelf Research, in press.

Moore, M.I., Visser, A.W., and Shirtcliffe, T.G.L. (1987). Experiences with the Brillinger spectral estimator applied to simulated irregularly observed processes. J. Time Series Anal., 8, 433-442.

Morettin, P.A., and de Mesquita, A.R. (1978). Métodos de análise espectral na oceanografica. Bolm. Inst. Oceanog. Sao Paulo, 27, 153-190.

Ogata, Y., and Katsura, K. (1988). Likelihood analysis of spatial inhomogeneity for marked point patterns. Ann. Inst. Statist. Math., 40, 29-39.

Priestley, M.B. (1963). Discussion of Professor Bartlett's paper. J. Roy. Statist. Soc. Ser. B, 25, 288-290.

Stoyan, D. (1984). On correlations of marked point processes. Math. Nachr., 116, 197-207.

Tufte, E.R. (1983). The Visual Display of Quantitative Information. Graphics Press, Cheshire.

Tukey, J.W. (1963). An introduction to the frequency analysis of time series. The Collected Works of John W. Tukey. Volume 1, 1984. (D.R. Brillinger, ed.). Wadsworth, Pacific Grove, Calif., 503-650.

Vale, R.D. (1993). Measuring single protein motors at work. Science, 260, 169-17.0.

Vere-Jones, D. (1970). Stochastic models for earthquake occurrence (with Discussion). J. Roy. Statist. Soc. Ser. $B, 32,1-62$.

Vere-Jones, D., and Davies, R.B. (1966). A statistical survey of earthquakes in the main seismic region of New Zealand. N.Z. J. Geol. Geophys., 9, 251-284.

Wartzok, D., Sayegh, S., Stone, H., Barchak, J., and Barnes, W. (1992). Acoustic tracking system for monitoring under-ice movements of polar seals. J. Acoust. Soc. Amer. 92, 682-687.

Watson-Watt, R. (1946). The evolution of radar location. J. Inst. Elect. Engrg., 93, 6-20.

Waters, K.H. (1978). Reflection Seismology. Wiley, New York.

Whittaker, E., and Robinson, G. (1944). The Calculus of Observations. Blackie, London.

Wood, F.J. (1978). Tidal Dynamics. Reidel, Dordrecht.

Received 19 August 1993

Revised 20 December 1993

Accepted 21 December 1993
Department of Statistics

University of California

Berkeley, California

U.S.A. 94720 